\title{
Benard Convection in a two Component System with Soret Effect
}

\author{
G. Zimmermann, U. Müller \\ Institut für Reaktorbauelemente
}

\section{Kernforschungszentrum Karlsruhe}





\title{
KERNFORSCHUNGSZENTRUM KARLSRUHE
}

\author{
Institut für Reaktorbauelemente
}

KfK 4860

Benard Convection in a two Component System with Soret Effect

G. Zimmermann, U. Müller

Kernforschungszentrum Karlsruhe $\mathrm{GmbH}$, Karlsruhe 
Als Manuskript vervielfältigt

Für diesen Bericht behalten wir uns alle Rechte vor

Kernforschungszentrum Karisruhe $\mathrm{GmbH}$

Postfach 3640,7500 Kartsruhe 1

ISSN 0303-4003 


\section{Benard convection in a two-component system with Soret effect}

\section{ABSTRACT}

Flow visualization experiments are reported which study Rayleigh-Benard convection in a two component system with Soret effect. Differential interferometry in combination with local temperature records inside the liquid layer is employed to analyse the spatial and temporal behavior of the convection in a slender test cell of dimension height:depth:width $=0.31: 2: 20$. Travelling roll patterns of transient and permanent character as well as steady overturning convection are observed depending on the range of Rayleigh numbers. The transitions from the static state of conduction to the travelling wave state and the transition from travelling waves to steady convection is affected by hysteresis. The frequencies associated with the travelling waves are determined and compared with predictions of linear stability analysis and interpreted in terms of solute transport and mixing along streamlines. The observations are in agreement with results reported in the literature. An unexpected feature is that the range of occurrence of the permanent state of travelling waves shrinks with a decreasing mean temperature of the liquid layer, and moreover disappears for mean temperatures below $5^{\circ} \mathrm{C}$. We attribute this effect to the increasing nonlinearity in the density-temperature relation at low temperatures.

\section{Benard Konvektion in einem Zwei-Komponenten-System mit Soret Effekt}

\section{ZUSAMMENFASSUNG}

Eine Rayleigh-Benard Konvektion wird in einer binären Flüssigkeit mit SoretEffekt untersucht, die sich in einem Rechteckbehälter mit den Abmessungen Höhe:Tiefe:Weite $=0.31: 2: 20$ befindet. Zur Analyse der räumlichen und zeitlichen Vorgänge werden ein Differential-Interferometer und Temperatursonden eingesetzt. Es werden stationäre Konvektionszustände und Zustände in Form rechts oder links wandernder Wellen beobachtet. Die mit den Wanderzuständen verknüpften Frequenzen werden mit Hilfe eines Mischungsmodells entlang Stromlinien erklärt.

Es zeigt sich, daß der Zustand der Wanderwellen verschwindet, wenn die mittlere Flüssigkeitstemperatur unter $5^{\circ} \mathrm{C}$ abfällt. Es wird vermutet, daß dieser Effekt durch die wachsende Nichtlinearität der Dichte-Temperaturbeziehung verursacht wird. 


\section{Inhaltsverzeichnis}

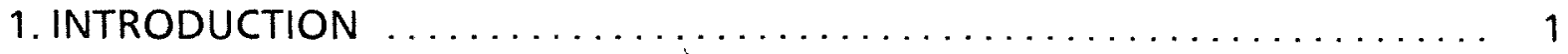

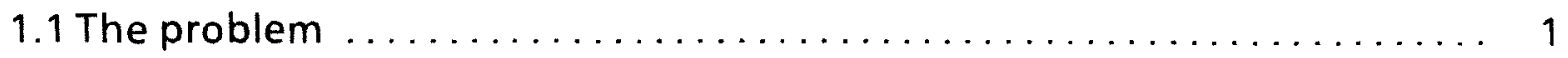

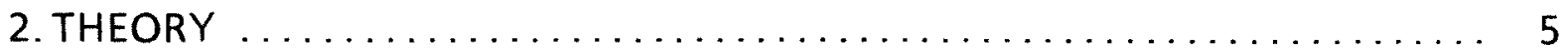

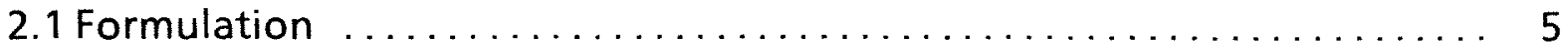

2.2 Differential Equations and Boundary Conditions $\ldots \ldots \ldots \ldots \ldots \ldots \ldots$

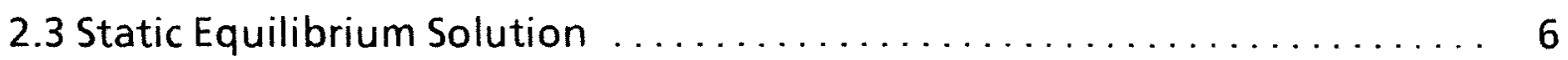

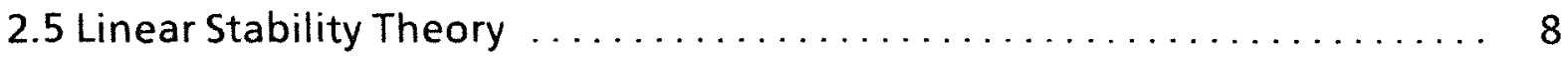

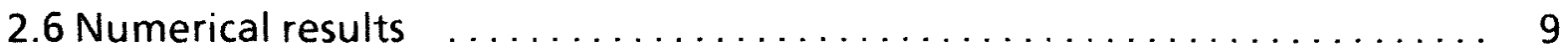

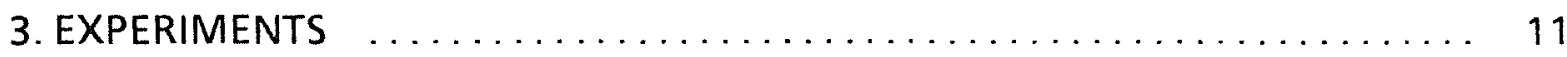

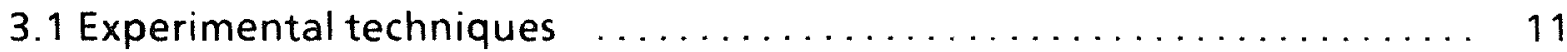

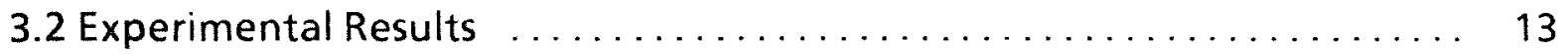

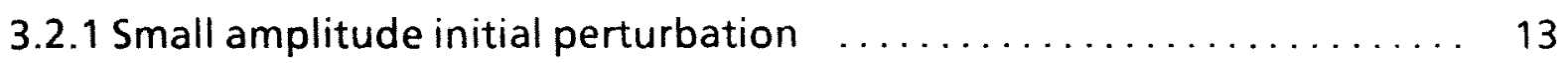

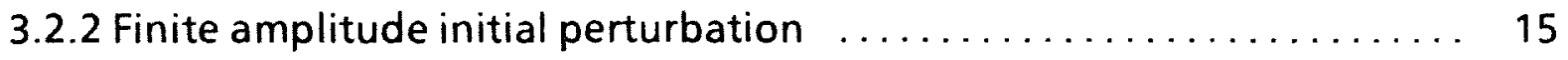

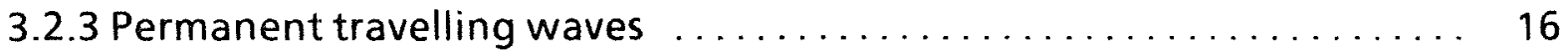

3.2.4 Transitions between permanent states $\ldots \ldots \ldots \ldots \ldots \ldots \ldots \ldots \ldots$

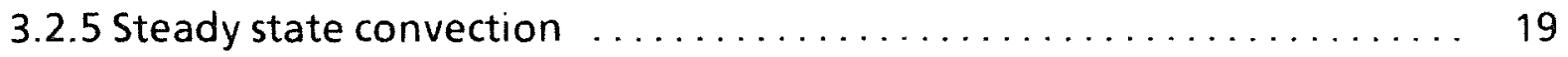

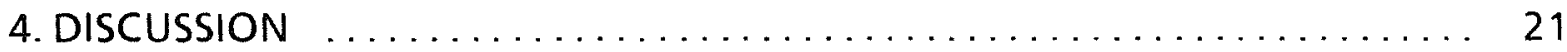

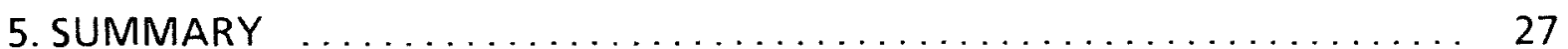




\section{INTRODUCTION}

\subsection{The problem}

Convective mass transport in the liquid phase may decisively determine the quality of technical materials when produced by controlled solidification. Typical examples are the growth of crystals from liquid solutions and precision casting of ingots using directional solidification. The convection in the liquid, usually initiated by inhomogeneities of an external temperature field, can change the local temperature and concentration field at the liquid/solid interface, which in turn may strongly effect a controlled solidification process and the solidified material.

A binary liquid mixture subject to temperature gradients generates thermal diffusion i.e. temperature gradients cause solute fluxes. This phenomenon is known as Soret Effect [Soret (1879), De Groot and Mazur (1969)]. The induced Soret solute flux may either add to or subtract from the imposed thermal gradient. In the latter case the opposing fields may generate oscillatory convective responses.

Single-phase convection in binary mixtures with negative Soret coefficient has been investigated intensively in the past two decades. Caldwell $(1970,1973$, 1974) studies Benard convection in sea water and finds a stabilizing effect of the thermal mass diffusion on the static state of heat conduction. By local temperature measurements within the layer he detects time-periodic temperature oscillations of small intensity when a critical condition for the static state is exceeded. For a fixed supercritical temperature difference the intensity and the period of oscillations increase monotonically in a transient phase until finally a permanent state of oscillatory convection is reached. Using Lithium iodine solutions Caldwell $(1975,1976)$ explores even smaller negative Soret coefficients and observes similar phenomena.

Hurle and Jakeman (1971) and Platten and Chavepeyer (1972a, b, 1973) perform experiments in alcohol-water mixtures. They do not observe transient oscillatory states of varying frequency but describe stable permanent states of oscillatory convection of constant frequency.

Alcohol-water mixtures have proven particularly easy to handle and have been utilized by numerous investigators (Walden et al. 1985, Kolodner et al. 1986, 1987a, b, Surko et al. 1986, Steinberg and Moses 1987, Steinberg, Moses and Fineberg 1987, Heinrichs et al. 1987, Ahlers, Cannell and Heinrichs 1987, Fineberg et al. 1988a, b, 1989, Bensimon et al. 1989) in their studies of the dynamics of convection in mixtures with Soret effects. In many of these recent experiments a lightscattering method has been employed in order to visualize the time dependent behaviour of the convection cell pattern. The convection pattern is visualized from 
above through a transparent upper boundary of the test cell by generating shadowgraphs of the light reflected from the mirror polished bottom of the test cell. The main results from these experiments can be summarized as follows:

- Immediately after the onset of a weak convection for slightly supercritical temperature differences across the layer, the roll pattern starts moving in the horizontal direction perpendicular to the roll axis. The convection presents itself as a travelling wave (Walden et al. 1985, Kolodner et al. 1986, Steinberg and Moses 1987, Moses et al. 1987). The amplitudes of the travelling waves may exhibit modulation. (Heinrichs et al. 1987, Fineberg et al. 1988a)

- The low intensity state of travelling waves is unstable. In a transient period the intensity of the convective motion increases while the propagation speed of the wave motion decreases.

- At the end of the transient period a stable, permanent state of travelling waves is observed characterized by a constant phase velocity (Surko et al. 1986, Moses and Steinberg 1986, Steinberg and Moses 1987).

- If the temperature difference across the layer is increased, the phase velocity of the stable state of travelling wave decreases. At another higher value of the temperature difference a smooth transition to steady convection, often called overturning convection occurs (Walden et al. 1985, Kolodner et al. 1986, Ahlers and Rehberg 1986, Rehberg and Ahlers 1986, Moses and Steinberg 1986, Steinberg and Moses 1987, Sullivan and Ahlers 1988a, b, Linz et al. 1988).

In part of the cited experimental work cells of small lateral extent were used. A clear cut picture of the various travelling wave effects is not always obtained mainly because of the uncontrolled generation of and the interaction of travelling wave patterns at different locations in the cell. This experimental deficiency is largely eliminated by the annular type test cell used by Kolodner et al. (1988 a) and Bensimon et al. (1989). Their test cell eliminates end effects in the azimuthal direction and so avoids azimuthal wave reflections. These experiments realize travelling wave packets in both azimuthal directions. They observe modulated travelling waves generated by the interaction of wave packets of slightly different wave length. They also find a permanent finite amplitude state of travelling waves either in confined regions of the annulus or of uniform intensity with regard to the azimuthal direction. Similar observations have been made by Heinrichs et al. (1987) and Ahlers et al. (1987) in rectangular test cells with one horizontal dimension much larger than the other and the cell height. Long range ma- 
terial transport due to wave action has been identified by Moses and Steinberg (1988) by adding fluorescent tracers to the liquid.

The stability of the static state of a binary mixture with Soret effect was first studied theoretically by Hurle and Jakeman $(1969,1971)$. Further investigations of this problem have been conducted by Platten (1971), Schechter et al. (1972), Legros et al. (1972) and Platten and Chavepeyer (1972a, b). These authors base their stability analysis on the simplifying assumption that the concentration profile of the static state is linear and the separation ratio, characterizing the Soret effect, is constant everywhere in the mixture. Only Chock and $\mathrm{Li}$ (1975) solve the linear stability problem for a complete set of balance equations, describing the transport processes in mixtures. The essential result of the linear stability analysis can be summarized in terms of the Soret coefficient $S$ as a measure for the intensity of thermal mass. There is a critical value $S^{*}$ of $S$ such that $S^{*}<0$. There are two cases that occur when the temperature difference across the layer exceeds a critical value. When $\mathrm{S}>\mathrm{S}^{*}$, the static state is replaced by a state of steady convection. When $\mathrm{S}<\mathrm{S}^{*}$, a state of oscillatory convection occurs. At $\mathrm{S}=\mathrm{S}^{*}$ both convective states merge at a codimension-two point.

Weakly nonlinear theories for finite amplitude convection in binary mixtures have recently been developed by Knobloch (1986a, b) Cross (1986a) and Ahlers and Lücke (1987). Although these authors assume stress free and solute permeable boundaries in their model equations they do predict some typical dynamical features like travelling waves, and modulated travelling waves, instability of standing waves and variation of the frequency with amplitude as observed in experiments. Linz et al. (1987) have improved an 8-mode Galerkin model by taking into account realistic boundary conditions for the solute, i.e. vanishing solute flux at the boundaries. Wave interaction has been included into a nonlinear analysis by Brand et al. (1986) and Brand and Steinberg (1984). They suggest that spatially irregular, oscillatory convection patterns observed in some experiments can be explained via a Benjamin-Feir instability. The effect of finite test cell geometry on travelling wave convection has been investigated numerically by Deane et al. (1988) and analytically by Cross (1986b, 1988). They predict confined wave states in the neighborhood of walls perpendicular to the direction of wave propagation as well as sloshing of wave packets within test cell. They explain this phenomenon in terms of a weakly nonlinear interaction of counter propagating waves due to reflection at vertical walls.

In spite of the encouraging results obtained by the cited authors and others from nonlinear analysis, many of the experimentally-observed phenomena can not be explained sufficiently well today. Some technically interesting aspects of con- 
vection in binary mixtures such as influence of temperature dependent physical properties of the mixture as for instance the nonlinear density-temperature relationship have not been investigated up to date. The experimental investigations reported in this article will support the findings of previous authors by employing different visualisation and measuring techniques and, moreover, will address the effect of a nonlinear density-temperature relationship on the oscillatory convection. The experiments are performed in making preparation for an investigation concerning the interaction of convection in binary mixtures and solidification. These investigations are reported in a succeeding paper. 


\section{THEORY}

\subsection{Formulation}

In the following we outline briefly the linear stability theory of Chock and Li (1975).

Consider the configuration sketched in fig. 1 where the horizontal parallel plates of infinite horizontal extent are separated by a distance $h$. The lower plate at $\mathrm{z}=0$ has the fixed temperature $\mathrm{T}=\mathrm{T}_{0}$ while the upper plate at $\mathrm{z}=\mathrm{h}$ has the fixed temperature $T=T_{1}$. The material between the plates is a two-component liquid. Gravity of magnitude $\mathrm{g}$ is downward, the layer is heated from below, $\mathrm{T}_{1}<\mathrm{T}_{0}$.

The material properties are the density $\rho_{0}$, the specific heat $c_{p}$, the thermal conductivity $\Lambda$, the thermometric conductivity $\mathrm{x}$ the kinematic viscosity $\mathrm{v}$ and the volume expansion coefficients $a$ and $a^{\prime}$ for thermal and concentration effects, respectively. The lean component has diffusivity $\mathrm{D}$ and Soret coefficient $\mathrm{S}_{0}$. The sign of $S_{0}$ is defined as usual in the literature (e.g. Legros and Platten (1977)). In the case of $\mathrm{S}_{0}>0$ the more dense component diffuses toward the upper, colder boundary. The velocity is $\mathrm{v}=(\mathrm{u}, \mathrm{v}, \mathrm{w})$.

\subsection{Differential Equations and Boundary Conditions}

The convection will be described using the Boussinesq equations. Therefore we have in the liquid, (see Chock and $\mathrm{Li}$ (1975)),

$$
\begin{gathered}
v_{t}+v \cdot \nabla v=-\frac{1}{\rho_{o}} \nabla p+v \nabla^{2} v+\left[a\left(T-T_{1}\right)+a^{\prime}\left(C-C_{0}\right)\right] g k \\
\nabla \cdot v=0 \\
T_{t}+v \cdot \nabla T=\mathrm{k} \nabla^{2} T \\
C_{t}+v \cdot \nabla C=-\nabla \cdot j, \\
\rho=\rho_{o}\left[1-a\left(T-T_{1}\right)-a^{\prime}\left(C-C_{o}\right] .\right.
\end{gathered}
$$

Here $\rho_{o}, \mathrm{C}_{0}$ are the mean density and mean mass fraction of the solute.

We use a linear equation of state (eq. 2.1 e) for the density in the buoyancy term of equation (2.1 a).

The flux j, allowing for Soret diffusion, takes the form 


$$
j=-D\left[\nabla C-S_{o} C(1-C) \nabla T\right]
$$

At the upper and lower boundary at $z=h$ and $z=0$ the temperatures are fixed and the boundaries are rigid. Thus we have:

$$
\begin{aligned}
& \mathrm{T}=\mathrm{T}_{1}, \mathrm{u}=\mathrm{v}=\mathrm{w}=0, \mathrm{z}=\mathrm{h} \\
& \mathrm{T}=\mathrm{T}_{\mathrm{o}}, \mathrm{u}=\mathrm{v}=\mathrm{w}=0, \mathrm{z}=0
\end{aligned}
$$

At the rigid boundaries the concentration flux vanishes.

$$
\mathrm{j} \cdot \mathrm{k}=0 \text { at } \mathrm{z}=0, \mathrm{z}=\mathrm{h}, \text { where } \mathrm{k}=(0,0,1)
$$

\subsection{Static Equilibrium Solution}

The governing system possesses a static equilibrium solution in which the velocity vector $\mathrm{v}$ is identically zero, the pressure $\mathrm{p}$ is hydrostatic and the temperatures are purely conductive. Here

$$
\bar{T}=T_{1}-\left(T_{o}-T_{1}\right) \frac{z-h}{h}
$$

Next we must find $\bar{C}$. We solve equations (2.1 d) and (2.2) subject to condition $(2.3 \mathrm{~b})$ and for given $\overline{\mathrm{T}}$ we find

$$
\bar{C}=\left\{1+\frac{1-\bar{C}(h)}{\bar{C}(h)} \exp \left[\frac{T_{o}-T_{1}}{h} S_{o}(z-h)\right]\right\}^{-1},
$$

where the constant of integration, the concentration $\bar{C}(h)$, is determined through the conservation law. We obtain from

$$
C_{o} h=\int_{o}^{h} C d z
$$

that

$$
\bar{C}(h)=\frac{\exp \left[\frac{\left(T_{0}-T_{1}\right)}{h} S_{o} h C_{0}\right]-1}{\exp \left[\frac{\left(T_{0}-T_{1}\right)}{h} S_{0} h\right]-1}
$$

The equations (2.1) and the boundary conditions (2.3) can be transformed into a dimensionless form by introducing the following scales 


$$
\begin{array}{ll}
\mathrm{x}, \mathrm{y}, \mathrm{z} & \sim \mathrm{h}, \\
\mathrm{t} & \sim \mathrm{h} 2 / \mathrm{k}, \\
\mathrm{u}, \mathrm{v}, \mathrm{w} & \sim \mathrm{k} / \mathrm{h}, \\
\mathrm{p} & \sim \rho_{\mathrm{o}} \mathrm{vk} / \mathrm{h}^{2}, \\
\mathrm{~T}-\mathrm{T}_{1} & \sim \mathrm{T}_{\mathrm{o}}-\mathrm{T}_{1}, \\
\mathrm{C} & \sim \mathrm{C}_{0} .
\end{array}
$$

The resulting dimensionless Boussinesq equations and interfacial conditions contain the following set of non-dimensional numbers.

$$
\begin{array}{ll}
R=\frac{\operatorname{ag}\left(T_{0}-T_{1}\right) h^{3}}{\mathrm{kv}}, & \text { Rayleigh number, thermal } \\
R_{s}=\frac{\mathrm{a}^{\prime} g h^{3}}{D v}, & \text { Rayleigh number, solutal } \\
P=\frac{\mathrm{v}}{\mathrm{K}}, & \text { Prandtl number } \\
S_{\mathrm{c}}=\frac{\mathrm{v}}{D}, & \text { Schmidt number } \\
S=S_{0}\left(T_{0}-T_{1}\right), & \text { Soret number } \\
C_{0} . & \text { Concentration, initial }
\end{array}
$$

The basic state $(2.4)-(2.6)$ can also be written in non-dimensional terms as follows:

$$
\begin{gathered}
T=1-z, \\
\bar{C}^{(L)}=\left\{C_{o}+\frac{1-C_{o} \bar{C}(1)}{\bar{C}(1)} \exp [S(1-z)]\right\}^{-1},
\end{gathered}
$$

where

$$
\bar{C}(1)=\frac{\exp \left[S C_{0}\right]-1}{\exp [S]-1} \frac{1}{C_{0}}
$$




\subsection{Linear Stability Theory}

We perturb the governing system (2.1) about the static basic state (2.4) - (2.6) introduce normal modes for each disturbance quantity $\phi^{\prime}$ as follows:

$$
\Phi^{\prime}(x, y, z, t)=\Phi e^{-\omega t+i\left(k_{1} x+k_{2} y\right)}
$$

where

$$
k=\left(k_{1}^{2}+k_{2}^{2}\right)^{1 / 2}
$$

and introduce the velocity components

$$
v^{\prime}=\left(u^{\prime}, v^{\prime}, w^{\prime}\right)
$$

and we denote $d / d z$ by $D$. We eliminate $u^{\prime}, v^{\prime}, p^{\prime}$ from the momentum equations by cross differentiation to obtain:

$$
\left(D^{2}-k^{2}\right)\left(D^{2}-k^{2}+\omega P^{-1}\right) W-k^{2}\left[R T+R_{s} P S_{c}^{-1} C_{o} C\right]=0
$$

From the heat transport equation and the transport equation for the lean component we get:

$$
\begin{gathered}
\left(D^{2}-k^{2}+\omega\right) T+W=0, \\
-D J-k^{2}\left[C-S \bar{C}\left(1-C_{o} \bar{C}\right) T\right]+\omega S_{c} P^{-1} C-S_{c} P^{-1} \bar{C}_{z} W=0,
\end{gathered}
$$

where

$$
-J=D C-S\left[\bar{C}\left(1-C_{o} \bar{C}\right) D T-\left(1-2 C_{o} \bar{C}\right) C\right] .
$$

The linearized boundary conditions for the ordinary differential equations (2.11) are

$$
\begin{aligned}
& \text { On } \mathrm{z}=0, \quad \mathrm{~T}=\mathrm{W}=\mathrm{DW}=\mathrm{J}_{3}=0 ; \\
& \text { On } \mathrm{z}=1, \quad \mathrm{~T}=\mathrm{W}=\mathrm{DW}=\mathrm{J}_{3}=0 .
\end{aligned}
$$

The boundary-eigenvalue problem defined by equations 2.11 and 2.12 is solved numerically by means of a shooting method. For this the SUPORE-CODE of Scott and Watts (1975) is used. 


\subsection{Numerical results}

The numerical results are obtained for a set of parameters typical for organic mixtures like cyclohexane-benzene. We use $\mathrm{P}=17.6, \mathrm{~S}_{\mathrm{c}}=1047, \mathrm{R}_{\mathrm{s}}{ }^{*}=28.4$. Calculations are also performed for ethyl alcohol-water mixtures with $\mathrm{C}_{0}=0,15$ $\left(C_{0}=0.08\right), P=30, S_{c}=8900 ; R_{S}^{*}=173.4$ and $\left.S=-0.019(S=-0.023) .{ }^{1}\right)$

We examine the pure Soret convection problem in which the layer is all liquid. Fig. 2 shows for $\mathrm{C}_{0}=0.01$, the critical Rayleigh number, the wave number and the oscillation frequency as functions of $S$.

When $\mathbf{S}=0$, there is no Soret effect and instability sets in as steady Benard convection with $R_{c}(s)=1708, k_{c}(s)=3.117$ and $\omega_{c}=0$. When $S>0$, the instability results in a steady state with, $\omega_{c}=0$, and both $R_{c}(s)$ and $k_{c}(s)$ decrease with $S$, consistent with the results of Chock and $\mathrm{Li}(1975)$. When $\mathrm{S}>0$, the Soret diffusion moves the more dense component toward the cold boundary reinforcing the adverse density gradient. When $\mathrm{S}<0$, the opposite is the case and the stationary instability is opposed, as shown in fig. 2 and in the results of Chock and Li (1975). In addition, when $S$ is sufficiently negative, $S<S^{*}<0$, a new mode, periodic in time, occurs. The corresponding critical Rayleigh number $R_{c}{ }^{(0)}$ is smaller than $R_{c}(s)$ for each $\mathrm{S}$. The $\mathbf{k}_{\mathrm{c}}{ }^{(0)}$ is slightly smaller than $\mathrm{k}_{\mathrm{c}}(\mathrm{s})$ while the frequency $\omega_{\mathrm{c}}$ of this oscillatory mode decreases with $S$ and $\omega_{c} \rightarrow 0$ as $S \rightarrow S^{*}$. Thus, the modes merge as $S \rightarrow S^{*}$. The value $S^{*}$ is in the literature commonly referred to as a codimension-two point. The present calculations give $S^{*}=-0.00025$. In general $R_{c}{ }^{(0)}, k_{c}{ }^{(0)}$ and $\omega_{c}$ all increase with $|S|$. Platten and Legros (1984) discuss how $S^{*}$ depends on $P, S_{c}$ and $R_{S}$.

1)

$$
R_{s}^{*}=\frac{\mathrm{a}^{\prime}}{\mathrm{a}} \frac{1}{T_{0}-T_{1}}
$$


We again examine the pure Soret convection problem but for different initial concentrations $\mathrm{C}_{0}$. Fig. 3 shows how $\mathrm{R}_{\mathfrak{c}}, \mathrm{k}_{\mathfrak{c}}$ and $\omega_{0}$ depend on $\mathrm{C}_{0}$. For conditions of stationary instability, $S>S^{*}$, all curves cross at $S=0$ where Soret diffusion is absent. $R_{c}(S)$ decreases with $C_{0}$ for $S>0$ as expected since more of the lean component is present to participate in Soret diffusion. Again, as expected, $\mathrm{k}_{\mathrm{c}}(\mathrm{s})$ decreases with $\mathrm{C}_{0}$ for fixed $\mathrm{S}>0$. For the condition of oscillatory instability $\mathrm{S}<\mathrm{S}^{*}$ for fixed $\mathrm{S}$ all the quantities $R_{c}{ }^{(0)}, k_{c}{ }^{(0)}$ and $\omega_{c}$ increase with $C_{0}$. For more detailed calculations see Zimmermann et al. 1986. They show that both $R_{c}{ }^{(0)}$ and $k_{c}{ }^{(0)}$ increase nearly linearly with $\mathrm{C}_{0}$. The above results are consistent with the idea that an increase in $\mathrm{C}_{0}$ effectively increases $\mathrm{S}$. As can be seen from eqns. $(2.11 \mathrm{c}, \mathrm{d})$, the Soret diffusion enters the governing equations through a term of the form $\mathrm{SC} \cdot(1-$ $\left.\mathrm{C}_{0} \overline{\mathrm{C}}\right)$. The numerical calculations show that $\overline{\mathrm{C}}(\mathrm{L})$ is nearly linear with $|\mathrm{d} \overline{\mathrm{C}} / \mathrm{dz}|<10$ 3. Thus, $\overline{\mathrm{C}} \sim \mathrm{C}_{0}$ and if $\mathrm{C}_{0} 2<<1$, then

$$
S \bar{C}\left(1-C_{o} \bar{C}\right) \approx S C_{o}=S_{e f f} .
$$

An increase of $C_{0}$ is equivalent to an increase in $|S|$, consistent with the results of fig. 3. Note that eqn. $(2.11 \mathrm{~d}$ ) has a second term dependent upon $\mathrm{S}$. Given the above argument, it becomes independent of $\mathrm{C}_{0}$. 


\section{EXPERIMENTS}

\subsection{Experimental techniques}

The experiments are performed in a test apparatus, which is similar to the one described by Dietsche \& Müller (1985), as sketched in fig. 4. A rectangular test volume of aspect ratio length: width: height $=200 \mathrm{~mm}: 20 \mathrm{~mm}: 3.12 \mathrm{~mm}$ is chosen in order to foster the generation of regular roll convection patterns. The volume is demarked by two copper blocks which serve as the upper and lower boundaries. Crystal glass plates form the long side walls. Two Teflon blocks are placed between the copper blocks and simultaneously serve as spacer elements and shorter side walls. O-rings are employed to seal off the cavity. The copper blocks are heated or cooled by circulating temperature-controlled coolant from thermostats through jackets at the upper and lower end of the two blocks. The overall design of the test apparatus is based on three-dimensional heat-conduction calculations that provide a very good approximation to a linear temperature distribution across the cavity in the preconvective state. According to calculations, horizontaltemperature inhomogeneities are less than $0,1 \%$ at the ends of the longsides and less than $0,01 \%$ otherwise. Nearly-ideal temperature conditions before onset of convection are needed to investigate the transition to oscillatory flow, since even the smallest convective motions, due to temperature inhomogeneities, may disturb significantly the concentration profile in the layer. The test apparatus is insulated externally by a styrofoam cover and further is placed in an air-conditioned chamber with good temperature control. The air temperature in this chamber is kept at the mean temperature of the test liquid during the experiments i.e. $T=\frac{1}{2}$ $\left(\mathrm{T}_{1}+\mathrm{T}_{0}\right)$. This procedure proved to be necessary for tests with solidification at temperatures significantly lower than the laboratory temperature which will be reported in a succeeding paper.

The selection of a suitable test liquid is crucial for a clear experimental identification of the phenomena. For our experiments the following requirements had to be met: a sufficiently negative value of the Soret coefficient; good transparency of the liquid for employing visualization techniques; near-ideal liquid solution with constant physical properties, a linear density-temperature and - concentration dependence (i.e. Boussinesq-properties), solidification of pure material (the solvent only) when the temperature falls below the liquidus temperature.

After thorough pretests employing cyclohexane-benzene mixtures and methyl alcohol-water mixtures the latter was chosen as a test liquid mainly because of its strong Soret effects. Alcohol-water mixtures have been used frequently in the past by experiments among others by Hurle and Jakeman (1971, 1973a, b), Villers and Platten (1984), Lhost and Platten (1988). The physical properties of this mix- 
ture are well documented in the literature. In particular the effective Soret number $\mathrm{S}$ eff which characterizes the thermal diffusion effect, can be varied between negative and positive values by changing the concentration of the solute (see Kolodner et al. 1988b) and the mean temperature in the test liquid. Moreover, only pure water is solidified from such dilute mixtures ..$^{\prime}$

Just as pure water has a density maximum at $4{ }^{\circ} \mathrm{C}$, low-concentration alcohol-water mixtures may have maxima near the solidification temperature $\mathrm{T}_{\mathrm{S}}$. They occur for alcohol concentrations $\mathrm{C} \leq 14 \mathrm{wt} \%$ but not otherwise, though even for $\mathrm{C}>14$ wt\% the mixture may still have nonlinear density profiles in the basic state (see Pickering 1893, De Coppet 1892). Higher solution concentration also shifts the operating point for negative Soret coefficient $\mathrm{S}$ towards $\mathrm{S}^{*}$, which gives oscillations of both low frequency and low intensity.

As a compromise, a mixture of 15 wt\% ethyl alcohol and water is used in most experiments. Some experiments are also carried out with mixtures of 8 wt\% ethyl alcohol in water. In these experiments dynamic phenomena without solidification are investigated and compared with results reported in the literature.

The quality of the measurement is determined by the long-term constancy of the temperatures at the upper and the lower boundary of the test cavity. These temperatures are controlled by two high-precision Haake thermostats of a coolant outlet-temperature-variance $\Delta \mathrm{T}= \pm 0.01^{\circ} \mathrm{C}$. At low temperatures ethyl alcohol is used as a coolant. The thermostats are connected to the test apparatus by plastic hoses isolated by thick rubber-foam wrappings.

The flow in the cooling channels of the test apparatus is counter current. The temperature in the copper blocks are measured by precision platinum resistance thermometers which are threaded through bore holes in the copper blocks very close to the boundaries of the liquid layer. The measuring arrangement used determines the temperature difference across the layer to an accuracy of $\Delta \mathrm{T}= \pm$ $0.002^{\circ} \mathrm{K}$. The quality of the temperature control of the test apparatus may be judged by the fact, that for static or for stable flow conditions in the liquid layer the measured temperature difference is constant up to $\delta(\Delta \mathrm{T})= \pm 0.003^{\circ} \mathrm{K}$ over time intervals of several days.

The temperature fluctuation within the liquid layer are measured by two $\mathrm{NiCr}-\mathrm{Ni}$ thermocouples of $0.25 \mathrm{~mm}$ in diameter protruding $0.9 \mathrm{~mm}$ into the test volume from the lower copper boundary. The thermocouple is located at the center of this boundary. The voltage between this thermocouple and a reference thermocouple in the lower copper is recorded. This voltage is amplified and further pro-

1) The requirement is relevant for experiments investigating the interaction of convection and solidification. Results of these investigations are reported in a succeeding paper. 
cessed to give signals of the temperature oscillations $\mathrm{T}^{\prime}$ in the test liquid as a pen chart record. The resolution of the temperature oscillations is $\delta \mathrm{T}^{\prime \prime}= \pm 0.02^{\circ} \mathrm{K}$ for the measuring arrangement used. From the chart records the period $\tau$ of periodic temperature fluctuation can be evaluated by averaging over a sequence of 20 periods to an accuracy of $\delta \mathrm{r} / \tau=0.01$.

The convective flow in the test volume was visualized by employing a differential interferometer (see Bühler et al. (1978), Kirchartz (1980) for an outline of the technique). In general the differential interferometer generates lines of constant density differences in direction of the beam separation. If the separation length $e$ is small as in the present case $(e=0.3 \mathrm{~mm})$, lines of constant density differences become lines of constant temperature gradients. Since the interferometer beams integrate the density differences only in one direction through the test volume, as is the case in the present set up, a quantitative evaluation of the fringe pattern is only possible for perfectly two dimensional flow. Since in experiments for certain conditions three-dimensional disturbances occur, we use the interferograms for only qualitative evaluation of the tests. In the present experiment only horizontal beam splitting is used. Bühler (1979) has shown for slightly supercritical conditions and two-dimensional flow that the fringe pattern generated from horizontal beam splitting can be interpreted to a good approximation as the stream line pattern. The interferograms are recorded by a high resolution video camera. Further processing is performed by employing a digital image processing system. Each interferogram shown in the figures of section 3.2 shows a segment of $43 \mathrm{~mm}$ of the total length of $200 \mathrm{~mm}$ of the test volume. In order to take interferograms from each part of the test volume the interferometer is placed on an optical bench which can be moved transversely in a controlled manner. When there are moving cellular patters, quantitative determination of the wave length and the direction and speed of the pattern movement can be made if one examines interferograms taken at distinct time intervals.

\subsection{Experimental Results}

\subsubsection{Small amplitude initial perturbation}

Experiments are conducted in a liquid mixture of $15 \mathrm{wt} \%$ ethyl alcohol in water at different mean temperatures $\overline{\mathrm{T}}=\frac{1}{2}\left(\mathrm{~T}_{0}+\mathrm{T}_{1}\right)$ of the layer. In a first set of tests the temperature difference across the layer is raised above its critical value for onset of time periodic flow by several small increments in time intervals $\Delta t$ of different duration.

Starting from a slightly subcritical stable static state of heat conduction the temperature difference is increased by about $\Delta \mathrm{T} \approx 0.1 \mathrm{~K}$ in a period of typically 30 
minutes. Thereby a weak convection is first stimulated at the ends of the test cell because of small temperature inhomogeneities due to end effects. New convection rolls form consecutively at the cell ends pushing the previously formed rolls towards the cell center and thus forming travelling wave trains. In general due to imperfections the cell formations at the cell ends do not occur symmetrically in time and space. Therefore usually the travelling wave originating from one of the two cell ends becomes dominant. When the waves travel across the thermocouple in the cell center, a periodic signal is recorded, whose amplitude is tine modulated. This convection mode is commonly denoted a modulated travelling wave (MTW). A typical record is shown in fig. 5. The maximum temperature amplitude has values $\mathrm{T}^{\prime \prime}=0.1 \mathrm{~K}$ and the period of oscillations is typically $\mathrm{\tau}=28 \mathrm{~s}$. The amplitude $\mathrm{T}$ " is small compared to the total temperature difference $\Delta \mathrm{T}=25.5 \mathrm{~K}$ across the layer. In general the intensity of the convection i.e. the amplitude of the temperature is amplified if the temperature difference $\Delta^{\prime} \mathrm{T}$ is kept fixed at a supercritical value.

When the temperature difference is reduced to its initial subcritical value a transition to the corresponding static state of heat conduction is observed as long as the amplitude of the convection has not exceeded a certain threshold value. This is also demonstrated in fig. 5 where the decrease of the amplitudes of oscillation for times $t>2.1 \cdot 10^{3} \mathrm{~s}$ is initiated by a reduction in the temperature difference at a time $t \approx 103$. The state of small amplitude modulated travelling waves can be maintained for practically unlimited time if the temperature difference across the layer is reduced to the critical value. The long time duration of the quasi-permanent modulated travelling wave state is demonstrated in fig. 6. A monotonic increase of $\Delta \mathrm{T}$ by $\delta \mathrm{T}=0.08 \mathrm{~K}$ during 30 minutes to slightly supercritical conditions of $\Delta \mathrm{T}=25.6 \mathrm{~K}$ results in a very weakly amplified travelling wave convection of period $\tau=28 \pm 0.2 \mathrm{~s}$ and a maximum amplitude $\mathrm{T}^{\prime}<0.05 \mathrm{~K}$ for many hours.

An increase of the temperature difference by $0.17 \mathrm{~K}$ at $\Delta \mathrm{T}=11.5 \mathrm{~K}$ within 30 minutes results in a stronger amplification of the modulated wave convection. When the amplitude finally exceeds a threshold value the modulated travelling waves settle down to a permanent state of travelling waves of constant amplitude for a particular fixed external temperature difference. This transition can be seen in fig. 7. The initially small fluctuation $\mathrm{T}^{\prime}$ increases within 20 minutes from $\mathrm{T}^{\prime \prime}= \pm 0.05 \mathrm{~K}$ at $\mathrm{t}=0$ to $\mathrm{T}^{\prime \prime}= \pm 0.5 \mathrm{~K}$ at $\mathrm{t}=2.5 \cdot 10^{3} \mathrm{~s}$ with a period of oscillation $\tau=37 \mathrm{~s}$. An optical impression of the MTW mode is obtained form the set of interferograms in fig. 8. 
The four interferograms show instantaneous convective states corresponding to the temperature record in fig. 7 near the center of the test cell at different times. In fig. 8a the lines of equal horizontal density gradients are only weakly displaced from the strict vertical direction. The interferogram corresponds to $t=210 \mathrm{~s}$. In figs. $8 \mathrm{~b}, \mathrm{c}, \mathrm{d}$ convective states of higher intensity are shown corresponding to times $t=1210 \mathrm{~s}, \mathrm{t}=1910 \mathrm{~s}$ and $\mathrm{t}=2230 \mathrm{~s}$. Bühler (1979) has shown, that the isolines of constant horizontal temperature gradients may be interpreted to a good approximation as a stream line pattern in the case of slightly transcritical twodimensional convection. Because of the slender geometry of the test cell and the quasi-steady heating, two dimensional convection is nearly established in the test volume with the axes of the travelling convection rolls parallel to the shorter side of the cell. The streamline pattern is sketched accordingly in fig. $8 \mathrm{e}$.

The feature of a travelling wave is displayed in fig. 9, where 14 interferograms taken at equal time intervals of $\Delta t=10 \mathrm{~s}$ are shown. The pattern moves from the left to the right side as is seen by the shift of the fringes with increasing time. The interferograms correspond to a time interval 1700s $\leq t \leq 1830$ s of the temperature record in fig. 7. The modulation of the intensity of the temperature perturbation due to convection can clearly be recognized from the number of concentric fringes which vary in the individual interferograms. More fringes indicate higher convection intensity in the travelling convection rolls. The period of oscillation of the travelling wave is $\tau=37 \mathrm{~s}$. This value is obtained from the temperature record in fig. 7 and also from the interferograms of fig. 9.

\subsubsection{Finite amplitude initial perturbation}

If the initial temperature ramp leading to a transcritical state exceeds a certain threshold value a zone of intensive finite amplitude convection is induced at the ends of the test cells. It turns out that this zone of intensive convection improves significantly the overall heat transfer. Since the heat supply is limited by the coolant flux through the lower copper-block of the test apparatus the temperature difference in the central range of the test section is slightly reduced to a subcritical value. As a result a perturbation front moves from the ends of the test cell towards the center. When this perturbation front moves across the thermocouple in the cell center a monotonically amplified periodic temperature signal is recorded whose amplitude after a short transition period reaches a saturation value. This is seen from the temperature record of fig. 10. In the corresponding experiment the temperature difference in the water ethyl alcohol mixture of $\mathrm{C}=8 \mathrm{wt} \%$ is raised within 2 minutes from $\Delta \mathrm{T}=6.88 \mathrm{~K}$ to $\Delta \mathrm{T}=6.98 \mathrm{~K}$. The experiment shows that the reverse procedure does not result in a subcritical static state rather 
the convective travelling wave state is maintained, however, at a slightly lower intensity. The proceeding perturbation front is depicted by the set of the interferograms of fig. 11. The interferograms are taken at time intervals $\Delta t=8 \mathrm{~s}$. The front propagates at the low speed of $\mathrm{V}_{\mathrm{f}}=1 \cdot 10^{-5} \mathrm{~m} / \mathrm{s}$.

The cellular fringe pattern indicates regular convection cells. In this particular case of strong initial perturbations the travelling waves propagate opposite to the perturbation front. Observations show that at the front new convection rolls are repeatedly created which then move at a higher speed towards the cell end. The thermocouple records a period of oscillation of $\tau=86 \mathrm{~s}$ which is by a factor of 3 larger than the value at onset of the convection. A schematic streamline pattern as conjectured from the interferograms is added in fig. 11.

Once the convection front reaches the opposite end of the test cell at a time $t=16.2 \cdot 10^{3} \mathrm{~s}$ the temporal character of the travelling wave changes once more. This can be seen in fig. 10. The thermocouple record shows in the time interval $18 \cdot 10^{3} \mathrm{~s}<\mathrm{t}<23 \cdot 10^{3} \mathrm{~s}$ an increase of the amplitude by $25 \%$ and a change in the period of oscillation from $\tau=71 \mathrm{~s}$ to $\tau=280 \mathrm{~s}$. This effect is attributed to the interaction of the original wave and a reflected wave originating from the other end of the test cell. The time period for the transition in the temperature record of fig. 10 is compatible with the propagation speed of the travelling waves obtained from interferograms and the dimensions of the test cell. The final state of the long period travelling waves is stable and persists for several days for fixed external temperature boundary conditions.

By choosing different temperature transients for an onset of convection in some experiments wave fronts are simultaneously initiated at both ends of the test cell. These wave fronts penetrate into the static layer in opposite directions. After merging into each other a permanent travelling wave of higher intensity and lower frequency is usually observed. However, for a low mean temperature $\overline{\mathrm{T}}=\frac{1}{2}\left(\mathrm{~T}_{0}+\mathrm{T}_{1}\right)$ of the liquid mixtures, say $\overline{\mathrm{T}} \leq 5^{\circ} \mathrm{C}$, for a $15 \mathrm{wt} \%$ ethyl alcohol water mixture a transitions to stable overturning convection occurs.

\subsubsection{Permanent travelling waves}

The permanent state of travelling waves in the test cell of finite length is investigated with regard to changing temperature differences $\Delta T$ across the liquid layer.

When $\Delta \mathrm{T}$ is increased the period of oscillation increases continuously until finally at a certain threshold temperature difference a jump transition occurs to steady overturning convection which is moreover observed for even higher temperature differences. The measurements show, that the oscillation frequencies de- 
pend linearly on the Rayleigh number in a range near the transition to steady overturning convection. A linear least quare fit of the frequency data gives the relations $\mathrm{f}=\mathrm{a}$ - $\mathrm{br}$ with $\mathrm{a}=16.5(\mathrm{mHz}), \mathrm{b}=9.8(\mathrm{mHz})$ for $\mathrm{C}_{0}=8 \mathrm{wt} \%$ and $\mathrm{a}=8.4$, $\mathrm{b}=5.2$, for $\mathrm{C}_{0}=15 \%$, where $\mathrm{r}$ is the reduced Rayleigh number $\mathrm{r}=\mathrm{R}_{\mathrm{c}}{ }^{(0)} / \mathrm{R}_{\mathrm{c}}$. If $\Delta \mathrm{T}$ is reduced starting from steady state convection travelling waves are observed only at a noticeably lower value $\Delta \mathrm{T}$ compared to the one where steady convection is first seen for increasing $\Delta T$. Thus, the transition from travelling waves to overturning convection and reverse shows a significant hysteresis. A similar phenomenon is observed for the onset of travelling wave motion from the static state. Decreasing $\Delta \mathrm{T}$ across the layer, the travelling wave mode persists to significantly lower values of $\Delta \mathrm{T}$ compared to its critical value for onset of travelling wave motion.

The range of existence of permanent travelling waves is explored for two mixtures of ethyl alcohol and water with $\mathrm{C}_{0}=8 \mathrm{wt} \%$ and $\mathrm{C}_{0}=15 \mathrm{wt} \%$. The experiments with these mixtures are carried out at mean temperatures $\overline{\mathrm{T}}=20^{\circ} \mathrm{C}$ and $\overline{\mathrm{T}}=10^{\circ} \mathrm{C}$ respectively. The results are given in figs. 12 and 13 in form of graphs showing the oscillation period as a function of the normalized Rayleigh number $r=R / R_{c}$ where $R_{c}=1708$ is the critical Rayleigh number for onset of convection in a single component liquid. Both mixtures show qualitatively the same behaviour. They differ, however, quantitatively in the measured periods and in the range where hysteretic behaviour is observed. This can be seen from table 1 where the transitions from the static state to the travelling wave and the steady state convection are listed.

For a mixture of $\mathrm{C}_{0}=15 \mathrm{wt} \%$ and a mean temperature $\overline{\mathrm{T}}=10^{\circ} \mathrm{C}$ the permanent travelling wave mode exists in the range $1.02 \leq \mathrm{r} \leq 1.59$ (see fig. 12 and table 1). Figures 14 and 15 show temperature fluctuations 'T" and a corresponding set of interferograms for three different Rayleigh numbers, $\mathrm{r}=1.10, \mathrm{r}=1.30$ and $r=1.49$. The temperature records as well as the set of interferograms show that irregularities in the temperature traces as well as in the fringe pattern of the interferograms occur at lower Rayleigh numbers but vanish at higher values (compare figs $14 \mathrm{a}$ and $14 \mathrm{~b}$ as well as figs. $15 \mathrm{a}$ and $15 \mathrm{~b} .{ }^{2}$ The short period variations in the frequency of ' $\mathrm{T}$ " occurring intermittently for $\mathrm{r}=1.10$ correspond to wave length variations between the individual moving convective rolls characterized by the concentric fringe patterns of the interferograms. By observations the temporal de-

2. Notice that the deformations in the fringes of interferograms of fig. $15 \mathrm{c}$ are due to strong light refraction in the thermal boundary layers at the lower and upper boundary for higher Rayleigh numbers. 
fects in the temperature record and the pattern irregularities do not seem to have a significant effect on the overall propagation speed of the wave motion.

The maximum value of the amplitude of the temperature fluctuation recorded by the thermocouple is plotted versus the normalized Rayleigh number in fig. 16 for a mixture of $\mathrm{C}_{0}=15 \mathrm{wt} \%$. This dependency can be to a good approximation, described by a linear function.

As outlined before, in a mixture of $\mathrm{C}_{0}=15 \mathrm{wt} \%$ ethyl alcohol and water, permanent travelling waves are observed in the range $1.02 \leq \mathrm{r} \leq 1.59$ if the mean temperature of the layer is $\overline{\mathrm{T}}=10^{\circ} \mathrm{C}$. If the mean temperature is lowered the range of existence for permanent travelling waves shrinks. For $\overline{\mathrm{T}} \leq 5^{\circ} \mathrm{C}$ permanent travelling waves do not occur. If in this case the temperature difference across the layer is increased beyond the critical value at $r=1.89$ a modulated travelling wave is first observed originating near one or the other end wall of the test cell. The amplitude of this wave is amplified to a finite value while propagating from one to the other side. Once the wave front reaches the opposite side wall the travelling wave motion is arrested and a steady overturning convection is established.

For a particular permanent state of travelling waves the end effects in the test cell are visualized in figs. 17 and 18. In fig. 17 the temperature gradient field in the vicinity of the end of the test cell is demonstrated by a set of interferograms taken at time intervals of $\Delta \mathrm{t}=90 \mathrm{~s}$. The interferograms depict a situation where the waves move away from the cell end. The temperature difference is in this case $\Delta \mathrm{T}=7.0 \mathrm{~K}$ and the initial concentration is $\mathrm{C}_{0}=15 \mathrm{wt} \%$. From the fringe pattern it can be concluded that heat conduction prevails in a range $0<\mathrm{L}<2 \mathrm{~h}$ (the interferograms show equidistant and vertically oriented fringes). In a transition range $\mathrm{h}<\mathrm{L} \leq 10 \mathrm{~h}$ the fringe increases continuously and the fringes become more and more contorted. The final permanent state indicated by concentric fringe patterns is established for $\mathrm{L}>10 \mathrm{~h}$. It can be observed that the wave propagation velocity develops in this range from zero to its final value.

Figure 18 shows the situation of a wave moving towards the end wall of the test cell. From the set of interferograms a change in the intensity of the wave mode and the propagation speed can not be identified in a region close to the end wall. However, a small decrease in the wave length of about $10 \%$ can be measured in a region $\mathrm{L} \leq 5 \mathrm{~h}$.

\subsubsection{Transitions between permanent states}

The dynamics of the transitions from the state of travelling waves to the static basic state and the state of overturning convection are demonstrated in the 
figs. 19, 20. When the Rayleigh number $r$ is reduced below $r=1.02$ the transition to the basic state of heat conduction occurs. This transition is a transient process starting at that end of the test cell where the travelling waves originate. The static state progresses from this side towards the other side of the test cell. The interferograms in fig. 19a demonstrate the retreat of the trailing edge of the wave and the progress of the static state. The observed velocity of the transition range is $v$ $=0.09 \mathrm{~mm} / \mathrm{s}$. The period length of the retreating waves is $\tau=68 \mathrm{~s}$. The width of the transition range is about 2-3 wave length. Figure $19 \mathrm{~b}$ gives the corresponding temperature record. The record shows that after the temperature difference is changed by $\Delta \mathrm{T}=0.04 \mathrm{~K}$ at $\mathrm{t}=0$ the amplitude of the temperature fluctuation is reduced in a modulated manner while the frequency increases. At a time $7200 \mathrm{~s}$ the last measurable temperature fluctuation passes the thermocouple. The period of oscillation lastly recorded by the thermocouple is $\mathrm{c}=67.9 \mathrm{~s}$. This value corresponds to the one evaluated from the interferograms in fig. 19a.

When the temperature difference is raised beyond the critical value for transition to steady overturning convection the period length of the temperature oscillation increases continuously until the wave pattern finally is arrested and the temperature signal at the thermocouple dies off. This is seen from the temperature record in fig. 20a obtained in a mixture of $\mathrm{C}_{0}=8 \mathrm{wt} \%$ ethyl alcohol and water. In this particular case a temperature rise of $\delta(\Delta T)=0.18 \mathrm{~K}$ occurs at $\mathrm{t}=0$. The ther . mocouple senses the steady state at $t=23 \cdot 10^{3} \mathrm{~s}$. The reverse phenomenon of $\mathrm{de}$ creasing periods of oscillation is observed during the transition from the steady state convection to the wave mode convection. This is seen in fig. $20 \mathrm{~b}$. In this case the temperature difference across the layer is decreased by $\delta(\Delta \mathrm{T})=0.3 \mathrm{~K}$ at time $t=0$. The permanent state of travelling waves with constant period length is achieved for $t \geq 2.2 \cdot 10^{4} \mathrm{~s}$. It has to be mentioned that the measurements in this range of parameters require long measuring times of, say, several days. Temperature constancy at the boundaries has to be maintained precisely (see Section 3.1).

\subsubsection{Steady state convection}

The experiments conducted at a mean temperature $\overline{\mathrm{T}}=10^{\circ} \mathrm{C}$ and in a mixture of $\mathrm{C}_{0}=15 \mathrm{wt} \%$ show that stable travelling waves exist in a range of normalized Rayleigh numbers $1.02 \leq \mathrm{r} \leq 1.59$. When the mean temperature of the liquid layer is reduced the range of existence of stable travelling waves becomes smaller and simultaneously the convection pattern gets less regular. For instance convection rolls of different wave length and different intensity occur. If the mean temperature $\overline{\mathrm{T}}$ falls below the threshold value $\overline{\mathrm{T}} \leq 5{ }^{\circ} \mathrm{C}$ permanent travelling waves are not observed. Thus, if in an experiment with $\overline{\mathrm{T}} \leq 5^{\circ} \mathrm{C}$ the temperature differ- 
ence across the layer is increased beyond its critical value to $r=1.89$, modulated travelling waves (MTW) are observed. This MTW state is amplified to a saturation amplitude. The saturated MTW propagates through the test cell usually starting at one end of the cell. Once this waves reaches the other end of the cell the velocity of the travelling wave slows down starting from this particular end. Finally an overturning steady state convection forms (see also Section 3.2.3). 


\section{DISCUSSION}

The occurrence of a permanent oscillatory convection in the layer consisting of a mixture with negative Soret coefficient is the result of a double- diffusive mechanism, in which the temperature and concentrations distribution have opposite stabilizing effects. Their spacial distribution diffuse with vastly different times scales $\tau_{T}$ and $\tau_{c}$ respectively. Since $\tau_{T} \ll \tau_{c}$, a warm fluid element that rises in the layer looses its characteristic temperature in time $\tau_{\mathrm{T}}$ while its characteristic concentration remains practically unchanged. Thus after equilibration of the temperature the parcel is too heavy compared to its environment, and falls back into a warmer layer. The decent of the parcel is on the longer time scale $\tau_{c}$ so the density adjusts to the temperature distribution. The parcel becomes lighter and rises again due to thermal buoyancy. The cycle starts anew. The above physical arguments are reasonable to explain the occurrence of an oscillatory convection in which the velocity in the convection cell changes sign for each half period. The experiments show, however, that this oscillatory state, which can be considered a standing wave, is not realized as a permanent state rather travelling roll patterns are observed.

The time and space dependent process in the layer can indeed be described mathematically as propagating waves of temperature, concentration and velocity. This has been done among others by Knobloch et al. (1986), Cross (1986a,b), A E . lers and Lücke (1987) and by Linz et al. (1988). In their weakly nonlinear analysis Linz and Lücke (1987) and Linz et al. (1988) discuss solutions of travelling waves (TW), modulated travelling waves (MTW), standing waves (SW) and overturning steady convection (OSC). They find for travelling waves a phase shift between the concentration, the velocity and the temperature fields. The phase shift between the temperature and concentration wave gives rise to a horizontal pressure variation along streamlines of a convection cell. This horizontal pressure variation makes the convection cells move in the preferred direction. The phase shift vanishes between the temperature and concentration field for standing waves. As a result there exists a net flux of the lean component in travelling wave convection whereas the time average flux vanishes for standing waves. A rather general result for wave propagation in double diffusive systems has been obtained by Knobloch et al. (1986). Applied to two component systems with Soret effect their analysis proves that near the critical Rayleigh number for onset of oscillatory convection the standing wave mode is unstable if a stable travelling wave mode bifurcates supercritically, and vice versa. If the bifurcation of the travelling wave occurs subcritically it is locally unstable and so is the related standing wave mode. 
For our experiments we observe modulated travelling waves, travelling waves and overturning convection. Permanent standing waves do not occur for the realized experimental conditions. The hysteresis effect related to the transition from the static state to the travelling wave mode as well as the sensitivity to finite amplitude perturbations in our experiments suggest that we have a subcritical bifurcation of the travelling wave mode which is thus locally unstable according to theory. The instability of the small amplitude travelling wave may also be concluded from the long term growth of the state variables, e.g. $T^{\prime}(t)$ in fig. 6 , until they settle down to a finite saturation value. This finite amplitude permanent travelling wave mode of the experiment is not described by the weakly nonlinear theory of Knobloch et al. (1986), Cross (1986) or Linz et al. (1988). However, Bensimon et al. (1989) have developed a nonlinear theory, which also describes the permanent travelling waves of finite amplitude and their transition to steady overturning convection.

Some features of the unstable modulated travelling waves of small amplitude are adequately described by the linear stability analysis of section 2 . In table 2 a comparison between measured and calculated values for the critical Rayleigh number and the period of oscillation is presented for mixtures of $\mathrm{C}_{0}=8 \mathrm{wt} \%$ and $\mathrm{C}_{0}=15 \mathrm{wt} \%$ ethyl alcohol and water and for different mean temperatures of the liquid layer. The approximate solution is obtained from analytical results of Hurle and Jakeman $(1969,1971)$ and from Kolodner et al. (1987b). According to these authors the following relations hold

$$
\begin{aligned}
& R_{c}^{(0)} \approx R_{c}\left(1-k_{3}\left|\frac{\Psi}{1+\Psi+\frac{1}{P r}}\right|\right) \\
& \omega_{c}^{(0)} \approx \frac{3 \pi^{2}}{2} k_{4}\left|\frac{-\Psi}{1+\Psi+\frac{1}{P r}}\right|^{1 / 2}
\end{aligned}
$$

with $\mathrm{k}_{3}=1.05$ and $\mathrm{k}_{4}=1.43$. Here $\Psi$ is the separation coefficient $\Psi=\mathrm{S} \mathrm{C}_{0}\left(1-\mathrm{C}_{0}\right)$ $a^{\prime} / a$. In the approximation a linear concentration profile in the static state and Le $\ll 1$ has been assumed.

It is seen, that the approximate values $R_{c}(0)$ and $\omega_{c}(0)$ agree well with the calculated ones by using the SUPORE-Code. There are differences between the calculated and the measured quantities in particular for the case $\overline{\mathrm{T}}=10^{\circ} \mathrm{C}$. These differences are attributed to uncertainties in the Soret coefficient. The value used in our evaluation is taken from Kolodner et al. (1988b). Generally the values found 
in the literature for S may differ up to $25 \%$. Other sources for the discrepancy may be the effectively nonlinear density temperature relation in mixtures and the finite extent of the test volume. Both effects are stabilizing and should give rise to higher critical values for $\mathrm{R}_{c}(0)$ and $\omega_{c}(0)$.

The recorded temperature fluctuation history of the travelling wave convection (see figs. 7, 10, 19, 20) has some features in common with the measured velocity history of Lhost and Platten (1989). These authors use LDV-technique to determine the vertical velocity component in the center of their test cell. In particular they find three distinct frequency ranges during the development of the wave propagation in the test cell. The initial state of relative high frequency as described by linear stability theory, a transient intermediate state of weakly nonlinear but still fast wave propagation and a final nonlinear permanent state of slowly travelling waves. The frequencies of these states differ typically by a factor $1 / 2$ and $1 / 10$ respectively. This finding agrees qualitatively with our observations of distinct frequency change to lower values when a) a threshold value of the amplitude of the travelling roll convection is exceeded and $b$ ) when the interaction of original and the reflected wave has occurred.

The progression of a perturbation front into an unstable static state with waves travelling in opposite directions, as demonstrated by fig. 11, can be compared with observations by Kolodner et al. (1988). These authors report confinet states of travelling waves resting or moving in an annular container. Depending on the direction of the waves in the moving confined region a similar situation must occur on the leading or trailing edge of such a wave packet. Kolodner (1990) reports an experiment in which slow travelling rolls exist in a confined region of an arbitrary length in a rectangular cell separated from the rest by a front that may slowly move or be motionless. A model to describe this phenomenon qualitatively has been proposed by Bensimon et al. (1988). The model is based on two coupled, generalized Landau Ginsburg equations. The model contributes physical explanations for the observed phenomena, but does not describe them quantitatively.

The influence of a finite geometry of the test cell on wave systems has been investigated by Moses et al. (1987), Surko and Kolodner (1987) and Ahlers et al. (1987). Cross $(1986,1988)$ investigates this question theoretically. He studies the reflection of waves at the vertical boundaries of test volumes using LandauGinsburg type amplitude equations. If the group velocity of the wave system is small the weakly nonlinear interaction of right and left side propagating waves results in a travelling wave state of preferred direction and uniform intensity in the major part of an horizontally elongated test cell. Only in the vicinity of the 
confining side walls does the interaction of the incoming and reflected wave lead to partial annihilation or the formation of a stationary convection depending on the direction of propagation of the wave in the cell center. Cross finds that the travelling wave is fully developed at a distance $\mathrm{L} \approx 10 \mathrm{~h}$ from the side wall where it originates; at the opposite side the impact of the wave on the vertical boundary is noticed at a distance of $L \approx 5 \mathrm{~h}$. These predictions are qualitatively confirmed by our observations as demonstrated by figs. 17 and 18. Our observations are in some qualitative agreement with the findings of Deane et al. (1988). They find for slightly supercritical Rayleigh numbers in their two-dimensional numerical simulation of binary convection in cells of large horizontal to vertical aspect ratio a nearly quiescent region near the one end and a small region of a standing wave pattern near the other end of the test cell, while in the cell center a travelling wave is predicted. Moreover, they predict an intermittent reversal of the direction of the travelling waves in the long run, which we do not observe.

The measured oscillation period of the permanent travelling wave state (see section 3.2.3) does not scale properly with either the thermal or the mass diffusion timescale $h 2 / k$ and $h 2 / D$ respectively. This seems plausible since this finite amplitude wave state is generally not near the bifurcation point for onset of oscillatory convection. Observations of tracer particles in the convective flow by means of a stereomicroscope indicate, that the turn over time is typically of the order $\tau_{\mathrm{t}} \approx 100 \mathrm{~s}$. The transport of the lean component within a moving convection roll (i.c. a travelling wave) can, to a good approximation, be described by the transport along closed streamlines in a coordinate system moving with the phase velocity of the wave. According to Rhines and Young (1983) this process can be described by the following transport equation

$$
\partial_{t} C+U \partial_{s} C=D \nabla^{2} C
$$

where $U$ is the transport velocity along a particular streamline, $s$ is the coordinate along the streamline and $\nabla 2$ is the two-dimensional Laplace operator in a corresponding orthogonal coordinate system. They obtain for a simple shear flow $U \sim$ ay a solution of the form

$$
C(t)=C_{0} \exp \left|-\left(\frac{t}{\tau_{D}}\right)-\left(\frac{t}{\tau_{m i x}}\right)^{3}\right| \cos k(s-\alpha y t)
$$

where $\tau_{D}=h^{2} / D$ and $\tau_{m i x}=\left(U^{2} D / h 4\right)^{-1 / 3}$. Introducing a Peclet number $P e=(U \cdot h / D)$ we obtain $\tau_{\text {mix }} \sim \mathrm{Pe}^{-1 / 3} \cdot \tau_{t}$. The form of the solution, eqn. (4.3), indicates that for 
large times the time scale $\tau_{\operatorname{mix}}$ determines the diffusion process in the vicinity of streamlines and thus the equalization of the concentration field within a moving convection cell.

Since the local Peclet number Pe and the turnaround time are directly related to the intensity of the convective flow and thus to the excess Rayleigh number $\Delta \mathrm{R}=\mathrm{R}-\mathrm{R}_{\mathrm{c}}(0)$, it is plausible that the transition from the travelling wave mode to steady overturning convection (see section 3.2.3) is due to an equalization of the concentration field by a transport-enhanced diffusion according to eqn. (4.3). We conjecture that $\tau_{\operatorname{mix}}$ is the proper time scale for the period of oscillation for the observed permanent travelling waves. Assuming the observed turn around time $\tau_{\mathrm{t}} \approx 10^{2} \mathrm{~s}$ and the dimension $\mathrm{h}=3 \mathrm{~mm}$ of our test volume and a typical diffusion coefficient $\mathrm{D} \approx 4 \cdot 10^{-4} \mathrm{~mm} 2 / \mathrm{s}$ we obtain a Peclet number $\mathrm{Pe} \approx 90$ and the corresponding mixing time scale $\tau_{\mathrm{mix}} \approx 450 \mathrm{~s}$. This value is of the order of the observed oscillation periods listed in figs. 12 and 13.

Our tests in mixtures of $\mathrm{C}_{0}=8 \mathrm{wt} \%$ and at mean temperature $\overline{\mathrm{T}}=20^{\circ} \mathrm{C}$ can be compared with experiments conducted by Walden et al. (1985) in a test cell of dimension height : width : length $=5.2 \mathrm{~mm}: 23 \mathrm{~mm}: 49 \mathrm{~mm}$ and for the same values of $\mathrm{C}_{0}$ and $\overline{\mathrm{T}}$. We observe similar hysteresis effects in our experiments as Walden at the transition from static conditions to travelling waves and from travelling waves to overturning convection. The different aspect ratio in the expe ments of Walden et al. results in a higher critical Rayleigh number for the onset 0 : wave motion. They obtain $r_{c}(0)=1.48$ compared to $r_{c}(0)=1.35$ in our case. Walden et al. find a state of permanent travelling waves in the range $1.17<\mathrm{r}<1.44$. In our slender test cell this state can be observed in the range $1.17<r<1.60$. It is conjectured that the discrepancy in the upper bounds of the ranges of existence are due to the three dimensional convective patterns occurring in the experiments of Walden et al. These authors measure oscillation periods of $1000 \mathrm{~s}<\tau<4000 \mathrm{~s}$ in a range $1.2 \leq \mathrm{r} \leq 1.44$. In our experiments periods in the range $240 \mathrm{~s} \leq \mathrm{\tau} \leq 900 \mathrm{~s}$ are found for $1.2 \leq \mathrm{r} \leq 1.54$. The differences can be related to the different dimensions of the test cells. If the periods in both experiments are scaled with the thermal diffusion time scale $\left(\tau_{T}=h^{2} / k\right)$ one obtains $4.6 \leq \hat{\tau} \leq 18.3$ for Walden's et al. experiment and $3.4 \leq \hat{\mathrm{\tau}} \leq 12.7$ in our case. This is a reasonable agreement reflecting the proper scaling of the geometry effect on $\tau_{\mathrm{T}}$.

The temperature records from the thermocouple in our test section show, that the generally periodic signal is intermittently disturbed by irregularities. They occur particularly for the shorter periods of oscillation and in the lower range of Rayleigh numbers, while at higher Rayleigh numbers and for longer periods such irregularities are not observed. Other authors refer to a similar observa- 
tion as chaotic behaviour which also involves pattern irregularities (see e.g. Walden et al. 1985, Steinberg, Moses 1987, Ahlers and Rehberg 1986). Our observations show that the temporal irregularities are related to irregularities in the wave number spectrum of the travelling waves (see figs. 15a, 15b). A complete theoretical model to explain this spatio-temporal feature is not available to our knowledge. A linear relationship between the frequency and the Rayleigh number (see section 3.2.3) as well as the hysteretic behaviour of the transition from the travelling wave mode to steady overturning convection has been observed by Moses and Steinberg (1986). It is also predicted in the theoretical works of Knobloch (1986a,b), Knobloch et al. (1986) and by the numerical simulations of Deane et al. (1987). It should be mentioned, however, that in the cited theoretical work unphysical boundary conditions, namely strees-free, and temperature and concentration fixed at the top and bottom, have been applied. It is conjectured, that the phenomena associated with the transition from TW to steady convection (homoclinic orbit) are generic and independent of boundary conditions.

Unexpectedly permanent travelling waves are not observed in ethyl alcoholmixtures if the average layer temperature is decreased to values that come close to the solidification temperature of the mixture, e.g. $\overline{\mathrm{T}}<5^{\circ} \mathrm{C}$ for a mixture of $\mathrm{C}_{0}=15 \%$. Instead of a travelling wave mode a steady three dimensional convection pattern develops. This effect is felt even for higher mean temperatures since the range, where permanent travelling waves are observed, shrinks when the average temperature is decreased. It is conjectured, that this effect is due to the increasing nonlinearity of the density-temperature relation for the mixture and is not related to the temperature dependence of the Soret coefficient (see Kolodner et al. (1988)). It is known that dilute salt-water mixtures exhibit a density anomaly at temperatures near the liquidus temperature $T_{L}$ of the mixture as water and ice does. It is also known that under these conditions the liquid layer is stabilized (see Veronis 1963). A theoretical analysis of the situation i.e. the effect of nonBoussinesq properties on wave propagation in binary mixtures is not available to date. 


\section{SUMMARY}

We have performed experiments of Benard convection in a binary mixture with Soret effect. We use a differential interferometer to visualize the convection patterns by interferograms and record the temperature history at a particular location inside the liquid layer. We observe an amplified travelling roll pattern of transient character whose intensity is modulated in space and time as long as its value is below a certain threshold. This travelling roll pattern starts at the shorter sidewalls. Once the value is exceeded the amplitudes of the convection saturate and a convection front propagates into the test cell. The convection fronts may start from each of the shorter side wall giving rise to an interaction of a pair of counter propagating travelling waves. This interaction may result in a standing wave pattern in the case of small supercritical Rayleigh numbers, or in the case of moderate supercritical Rayleigh numbers in a permanent travelling wave of a reduced phase velocity. In the later case the wave of the higher intensity determines the direction of propagation. There is a transition from the state of travelling waves to steady overturning convection for an even higher threshold Rayleigh number. This transition as well as the transition from the static state of heat conduction to the state of travelling wave convection show strong hysteresis effects. Permanent travelling waves are suppressed for low mean temperatures of the liquid layer. Instead a quasi-steady three-dimensional irregular convection pattern is identified. It is conjectured, that this phenomenon is due to the actual nonlinear density-temperature relation of the binary mixture at temperatures close to the liquidus temperature. The outlined observations are generally in agreement with results reported in the literature. 
G. Ahlers, D.S. Cannell, R. Heinrichs; 1987 :

Convection in a binary mixture; Nuclear Physics B (Proc. Suppl.) 2. 77-86.

G. Ahlers, M. Lücke; 1987:

Some properties of an eight-mode Lorenz model for convection in binary fluids; Phys. Rev. A35, 470-473.

G. Ahlers, I. Rehberg; 1986:

Convection in a Binary Mixture Heated from Below; Phys. Rev. Lett. 56, 1373 1376.

D. Bension, B. J. Shraiman, V. Croquette; 1988;

Nonadiabatic effects in convection; Phys. Rev. A38, 5461-5464.

D. Bensimon, A. Pumir, B. J. Shraiman; 1989

J. Physique 50, 3089 (1989)

D. Bensimon, P. Kolodner, C.M. Surko, H. Williams, V. Croquette; 1990 :

Competing and coexisting dynamical states of travelling-wave convection in an annulus. J.Fluid Mech. , 217, 441-67

H.R. Brand, P.S. Lomdahl, A.C. Newell; 1986:

Benjamin-Feir Turbulence in Convective Binary Fluid Mixtures; Physica 23D, 345361.

H.R. Brand, V. Steinberg; 1984:

Analog of the Benjamin-Feir instability near the onset of convection in binary fluid mixtures, Phys. Rev. A29, 2303-2304.

K. Bühler, 1979:

Zellularkonvektion in rotierenden Behältern; Fortschritt-Berichte der

VDI-Zeitschriften, Reihe 7, Nr. 54.

K. Bühler, K.R. Kirchartz, J. Srulijes; 1978:

Anwendung der Differentialinterferometrie bei thermischen

Konvektionsströmungen, in: Applied Fluid Mechanics; H. Oertel jr., Festschrift

Universität Karlsruhe, S. 54-69.

D.R. Caldwell; 1970 :

Nonlinear Effects in a Rayleigh-Bénard Experiment; J.Fluid Mech. 42, 161-175.

D.R. Caldwell; 1973:

Measurement of Negative Thermal Diffusion Coefficients by Observing the Onset of Thermohaline Convection; J.Phys. Chem. 77, 2004-2008.

D.R. Caldwell; 1974:

Experimental studies on the onset of thermohaline convection; J.Fluid Mech. $\underline{64}$, 347-367.

D.R. Caldwell; 1975 :

Sorét-Coefficient of $1 \mathrm{~N}$ Lithium Jodide; J.Phys.Chem. 79 , 1882-1884.

D.R. Caldwell; 1976:

Thermosolutal convection in a solution with large negative Sorét coefficient; J.Fluid Mech. 74, 129-142. 
D.P. Chock, Chin-Hsiu Li; 1975:

Direct Integration Method applied to Sorét Driven Instability; Phys. Fluids 18 , 1401-1406.

M.C. Cross; 1986a:

An eight-mode Lorenz-Model of travelling waves in a binary fluid convection; Phys. Lett. A119, 21-24.

M.C. Cross; 1986b:

Travling and Standing waves in Binary-Fluid Convection in Finite Geometries; Phys. Rev. Lett. 57, 2935-2938.

M.C. Cross; 1988 :

Structure of Nonlinear Travelling-Wave States in Finite Geometries; Phys. Rev. A38, 3593-3600.

A.E. Deane, E. Knobloch, J. Toomre; 1987:

Travelling Waves and Chaos in the Thermosolutal Convection; Phys. Rev. A36, 2862-2869.

A.E. Deane, E. Knobloch, J. Toomre; 1988:

Travelling waves in large-aspect-ratio thermosolutal convection; Phys. Rev. A37, 1817-1820 und Erratum: Phys. Rev. A38, 1661 (1988).

M. L. DeCoppet; 1892:

Sur la température du maximum de densité des mélanges d'al cool det d'eau;

Compt. rendu $115,652-653$.

S.R. DeGroot, P. Mazur; 1969:

Non-Equilibrium Thermodynamics; North-Holland Publishing Company, Amsterdam-London, 2nd Reprint.

C. Dietsche, U. Müller; 1985 :

Influence of Bénard convection on solid-liquid interfaces; J.Fluid Mech. 161, 249-268.

J. Fineberg, E. Moses, V. Steinberg; 1988a:

Spatially and Temporally Modulated Travelling-Wave Pattern in Convecting Binary Mixtures; Phys. Rev. Lett. 61, 838-841.

J. Fineberg, E. Moses, V. Steinberg; 1988b:

Nonlinear Pattern and Wave-Number Selection on Convecting Binary Mixtures; Phys. Rev. A38, 4939-4942.

J. Fineberg, E. Moses, V. Steinberg; 1989:

Reply to: P. Kolodner (1989); Phys. Rev. Lett. 64, 579.

R. Heinrichs, G. Ahlers, D.S. Cannell; 1987:

Travelling waves and spatial variations in the convection of a binary mixture; Phys. Rev. A35, 2761-2764.

D.T.J. Hurle, E. Jakeman; 1969:

Significance of the Sorét-Effect in the Rayleigh-Jeffreys' Problem; Phys. Fluids 12 , 2704-2705.

D.T. Hurle, E. Jakeman; 1971:

Sorét-driven thermosolutal convection; J.Fluid Mech. 47, 667-687. 
D.T.J. Hurle, E. Jakeman; 1973a:

Thermal Oscillations in Convecting Fluids; Phys. Fluids 16, 2056-2059.

D.T.J. Hurle, E. Jakeman; 1973b:

Natural oscillations in heated fluid layers; Phys. Lett. 43A, 127-129.

K.-R. Kirchartz; 1980:

Zeitabhängige Zellularkonvektion in horizontalen und geneigten Behältern;

Dissertation an der Universität Karlsruhe.

E. Knobloch; 1986a:

On the Degenerate Hopf-Bifurcation with O(2) Symmetry; Contemp. Math. 56 , 193-201.

E. Knobloch; 1986b:

Oscillatory Convection in Binary Mixtures; Phys. Rev. A34, 1538-1549.

E. Knobloch; A.E. Deane, J. Toomre, D.R. Moore; 1986:

Doubly diffusive waves; Contemp. Math. $\underline{56}$, 203-216.

P. Kolodner, A. Passner, C.M. Surko, R.W. Walden; 1986:

Onset of oscillatory convection in a binary fluid mixture; Phys. Rev. Lett. 56, 2621 2624.

P. Kolodner, A. Passner, H.L. Williams, C.M. Surko; 1987a:

The transition to finite-amplitude travelling-wave convection in binary fluid mixtures; Nuclear Physics B (Proc. Suppl.) 2, 97-108.

P. Kolodner, C.M. Surko, A. Passner; H.L. Williams; 1987b:

Pulses of oscillatory convection; Phys. Rev. A36, 2499-2502.

P. Kolodner, D. Bensimon, C.M. Surko; 1988a:

Travelling wave convection in an annulus; Phys. Rev. Lett. 60, 1723-1726.

P. Kolodner, H. Williams, C. Moe; 1988b:

Optical measurement of the Sorét coefficient of ethanol/water solutions;

J.Chem.Phys. 88, 6512-6524.

P. Kolodner; 1990;

Neutrally stable fronts of slow convective travelling waves; Phys. Rev. A42, 24752478.

J. C. Legros, J. K. Platten; 1977:

Two Component Bénard Problem with Poiseuille Flow; J. Non-Equilibrium Thermodynamics 2 , 211-232.

J.C. Legros, J.K. Platten, P.G. Poty; 1972:

Stability of a Two-Component Fluid Layer Heated from Below; Phys. Fluids 15 , 1383-1390.

O. Lhost, J.K. Platten; 1988:

Transitions Between Steady States, Travelling Waves, and Modulated Travelling Waves in the System Water-Isopropanol Heated from Below; Phys. Rev. A38, 3147-3150. 
O. Lhost, J.K. Platten; 1989:

Experimental study of the transition from nonlinear travelling waves to steady overturning convection in binary mixtures; Phys. Rev. A40, 4552-4557.

S.J. Linz, M. Lücke; 1987:

Convection in binary mixtures: A Galerkin model with impermeable boundary conditions; Phys. Rev. A35, 3997-4000 und Erratum: Phys. Rev. A38, 2486 (1988).

S.J. Linz, M. Lücke, H.W. Müller, J. Niederländer; 1988:

Convection in Binary Fluid Mixtures: Travelig Waves and Lateral Currents; Phys. Rev. A38, 5727-5741.

E. Moses, V. Steinberg, 1986:

Flow patterns and nonlinear behavior of travelling waves in a convective binary fluid; Phys. Rev. A34, 693-96 und Erratum: Phys. Rev. A35, 1444-1445 (1987).

E. Moses, V. Steinberg, 1988:

Mass transfer in propagating patterns of convection; Phys. Rev. Lett. $\underline{60}, 2030-$ 2033.

E. Moses, J. Fineberg, V. Steinberg, 1987:

Multistability and confined travelling-wave patterns in a convecting binary mixture; Phys. Rev. A35, 2757-2760.

S. U. Pickering; 1893:

A study of the properties of some strong solutions;

J. of the Chemical Society Transactions, Vol. LXIII, 998-1027.

J.K. Platten; 1971:

Le problème de Bénard dans les Mélanges: cas de surface libres; Bull. Classe Sci. Acad. Roy. Belg. 57, 669-683.

J.K. Platten, G. Chavepeyer; 1972a:

Oscillations in a water-ethanol liquid layer heated from below; Phys. Lett. 40A, 287-288.

J. K. Platten, G. Chavepeyer; 1972 b:

Soret Driven Instability; Phys. Fluids 15, 1555-1557.

J.K. Platten, G. Chavepeyer; 1973:

Oscillatory montion in Benard cell due to Soret effect;

J. Fluid. Mech. 60, 305-319.

J.K. Platten, J.C. Legros; 1984 :

Convection in Liquids; Springer Verlag, Berlin.

I. Rehberg, G. Ahlers; 1986:

Codimension-Two Bifurcation in a convection experiment; Contemp. Math. 56 , 277-282.

P.B. Rhines, W.R. Young; 1983:

How rapidly is a passive scalar mixed within closed streamlines? J. Fluid Mech. $133,133-145$.

R.S. Schechter, I. Prigogine, J.R. Hamm; 1972:

Thermal Diffusion and Convective Stability; Phys. Fluids 15 , 379-386. 
M.R. Scott, H.A. Watts; 1975 :

Subroutine SUPORE, Applied Mathematics Division 2623, Sandia Laboratories, Rep. SAND 75-0198, Albuquerque, NM.

M.Ch. Sorét; 1879 :

Une dissolution saline primitivement homogène; Arch. Sci. Phys. nat., Geneve, Vol. 2, 48-61.

V. Steinberg, E. Moses; 1987:

Experiments on convection in binary mixtures, in: Patterns, Defects and Microstructures in Nonequilibrium Systems; Proc. NATO Advanced Research Workshop; ed.: D. Walgraef, NATO ASI Series E, Martinus Nijhoff Publishers; Dordrecht, Boston; S. 309-335.

V. Steinberg, E. Moses, J. Fineberg; 1987:

Spatio-temporal complexity at the onset of convection in a binary fluid; Nuclear Physics B (Proc. Suppl.) 2 , 109-124.

T.S. Sullivan, G. Ahlers; 1988a:

Hopf Bifurcation to Convection near the Codimension-Two-Point in a $3 \mathrm{He}-4 \mathrm{He}-$ Mixture; Phys. Rev. Lett. 61, 78-81.

T.S. Sullivan, G. Ahlers; 1988b:

Nonperiodic Time Dependence at the Onset of Convection in a Binary Liquid Mixture; Phys. Rev. A38, 3143-3146.

C.M. Surko, P. Kolodner, A. Passner, R.W. Walden; 1986:

Finite-amplitude travelling-wave convection in binary fluid mixtures; Physica 23D, 220-229.

C. M. Surko, P. Kolodner; 1987:

Oscillatory Travelling-Wave Convection in a Finite Container

Phys. Rev. Lett. 58, 2055-2058

G. Veronis; 1963:

Penetrative convection; Astrophys. J. 137, 641-663.

D. Villers, J.K. Platten; 1984:

Heating curves in the two-component Bénard problem; J. Non-Equilibrium Thermodyn. $\underline{9}, 131-146$.

R.W. Walden, P. Kolodner, A. Passner, C.M. Surko; 1985:

Travelling waves and chaos in convection in binary fluid mixtures; Phys. Rev. Lett. 55, 496-499.

G. Zimmermann, U. Müller, S.H. Davis; 1986:

Bénard convection in a partly solidified two-component system; KfK-Bericht 4122. 
FIGURE CAPTIONS

Fig. 1: Schematic drawing of the liquid layer and the temperature and concentration profiles of the static state.

Fig. 2: Critical Rayleigh number $\mathrm{R}_{\mathfrak{c}}$, critical wave number $\mathbf{k}_{\mathfrak{c}}$, critical angular frequency $\omega_{\mathcal{c}}$ as a function of the Soret-number $S$ for an initial concentration $\mathrm{C}_{0}=0.01$. $\mathrm{S}^{*}$ denotes a codimension-two point.

Fig. 3: Critical Rayleigh-number, critical wave number $k_{c}$ and angular frequency $\omega_{c}$ as a function of the Soret-number $S$ for the initial concentrations $\mathrm{C}_{0}=0.01$ (solid line) and $\mathrm{C}_{0}=0.02$ (dashed line).

Fig. 4: Cross-section of the test apparatus.

Fig. 5: Local temperature fluctuations $\mathrm{T}^{\prime}$ recorded by the thermocouple in the cell center, $\mathrm{C}_{0}=15 \mathrm{wt} \%, \tilde{\mathrm{T}}=4.8^{\circ} \mathrm{C}$.

Fig. 6: Slowly amplified local temperature fluctuations $\mathrm{T}$ ' recorded by the thermocouple in the cell center, $\mathrm{C}_{0}=15 \mathrm{wt} \%, \overline{\mathrm{T}}=4.8^{\circ} \mathrm{C}$.

Fig. 7: Amplified local temperature fluctuations indicating the transition from a MTW- to a TW-mode, $\mathrm{C}_{0}=15 \mathrm{wt} \%, \overline{\mathrm{T}}=10^{\circ} \mathrm{C}$.

Fig. 8: Differential interferograms of an intermediate MTW-mode (see fig. 7) at times a) $t=210 \mathrm{~s}, \mathrm{~b}) \mathrm{t}=1210 \mathrm{~s}, \mathrm{c}) \mathrm{t}=1910 \mathrm{~s}, \mathrm{~d}) \mathrm{t}=2230 \mathrm{~s}$ and $\mathrm{e}$ ) schematic instantaneous streamline pattern.

Fig. 9: Differential interferograms of a MTW at equal time intervals $\Delta \mathrm{t}=10 \mathrm{~s}$ and $\mathrm{C}_{0}=15 \mathrm{wt} \%, \overline{\mathrm{T}}=10^{\circ} \mathrm{C}$ (see Fig. 7).

Fig. 10: Local temperature fluctuations $T "$ indicating onset of travelling waves due to finite amplitude initial perturbations $\left(t \geq 9 \cdot 10^{3} \mathrm{~s}\right)$ and transition to a stable permanent TW-mode $\left(t \geq 21 \cdot 10^{3} \mathrm{~s}\right) . \mathrm{C}_{0}=8 \mathrm{wt} \%$, $\overline{\mathrm{T}}=20^{\circ} \mathrm{C}$. 
Fig. 11: Differential interferograms and streamline patterns (schematic) demonstrating a progressing perturbation front and waves travelling in opposite direction. Time intervals between interferograms $\Delta \mathrm{t}=8 \mathrm{~s}$; $\mathrm{C}_{0}=15 \mathrm{wt} \%, \overline{\mathrm{T}}=10^{\circ} \mathrm{C}$.

Fig. 12: Period of oscillation $\tau$ of the permanent TW-state as a function of the normalized Rayleigh number $\mathrm{r}, \mathrm{C}_{0}=8 \mathrm{wt} \%, \overline{\mathrm{T}}=20^{\circ} \mathrm{C}$.

Fig. 13: Period of oscillation $\mathrm{t}$ of the permanent TW-state as a function of the normalized Rayleigh number $\mathrm{r}, \mathrm{C}_{0}=15 \mathrm{wt} \%, \overline{\mathrm{T}}=10^{\circ} \mathrm{C}$.

Fig. 14: Local temperature fluctuations T" recorded by the thermocouple in the cell center for the permanent TW-mode, $\mathrm{C}_{0}=15 \mathrm{wt} \%, \overline{\mathrm{T}}=10^{\circ} \mathrm{C}$ : a) $\mathrm{r}$ $=1.10, \tau=240 \mathrm{~s}$; b) $\mathrm{r}=1.30, \tau=654 \mathrm{~s} ; \mathrm{c}) \mathrm{r}=1.49, \tau=1550 \mathrm{~s}$.

Fig. 15: Differential interferograms corresponding to the temperature records of fig. 14, time intervals between interferograms: a) $\Delta t=60 \mathrm{~s}$, b) $\Delta t=$ $240 \mathrm{~s}, \mathrm{c}) \Delta \mathrm{t}=360 \mathrm{~s}$.

Fig. 16: Maximum amplitude of the temperature fluctuation T'm recorded $b y$ the thermocouple in the cell center as a function of the normalized Rayleigh number for the permanent TW-state. $\mathrm{C}_{0}=15 \mathrm{wt} \%, \overline{\mathrm{T}}=10^{\circ} \mathrm{C}$.

Fig. 17 Differential interferograms showing a TW-state near the end of the test cell. The TW moves toward the cell center. Time interval between interferograms $\Delta t=90 \mathrm{~s} ; \mathrm{C}_{0}=15 \mathrm{wt} \%, \overline{\mathrm{T}}=10^{\circ} \mathrm{C}$.

Fig. 18 Differential interferograms showing a TW-state near the end of the test cell. The TW moves toward the side wall, time interval between interferograms $\Delta \mathrm{t}=60 \mathrm{~s} ; \mathrm{C}_{0}=15 \mathrm{wt} \%, \overline{\mathrm{T}}=10^{\circ} \mathrm{C}$.

Fig. 19: Transition from TW-state to static state of heat conduction; a) differential interferograms show retreat of convective state; time interval between interferograms $\Delta \mathrm{t}=20 \mathrm{~s} ; \mathrm{C}_{0}=15 \mathrm{wt} \%, \overline{\mathrm{T}}=10^{\circ} \mathrm{C} ; \mathrm{b}$ ) local temperature. 
Fig. 20: Local temperature fluctuation recorded by the thermocouple in the cell center demonstrating a) the transition from a TW-state to overturning convection and $b$ ) the reverse process. 


\section{TABLES}

Tab. 1: Critical Rayleigh numbers for the transitions static state TW-state, TW-state OSC-state.

Tab. 2: Critical Rayleigh numbers and dimensionless periods of oscillation, comparison between theory and experiment. 


\begin{tabular}{|cccc|}
\hline Transition & $\begin{array}{c}\mathrm{C}_{0}=8 \mathrm{wt} \% \\
\overline{\mathrm{T}}=20^{\circ} \mathrm{C}\end{array}$ & $\begin{array}{c}\mathrm{C}_{0}=15 \mathrm{wt} \% \\
\overline{\mathrm{T}}=10^{\circ} \mathrm{C}\end{array}$ \\
\hline $\mathrm{r} \uparrow$ & 1,35 & 1,27 \\
& Heat Conduction $\rightarrow \mathrm{TW}$ & 1,60 & 1,59 \\
\hline $\mathrm{TW} \rightarrow$ Steady Convection & 1,54 & 1,42 \\
& Steady Convection $\rightarrow \mathrm{TW}$ & 1,17 & 1,02 \\
\hline
\end{tabular}

Table 1.

\begin{tabular}{|c|c|c|c|c|}
\hline & \multirow{2}{*}{$\frac{\text { Concentration }}{\text { Temperature }}$} & \multirow{2}{*}{$\frac{8 w t \%}{20^{\circ} \mathrm{C}}$} & \multicolumn{2}{|c|}{$15 w t \%$} \\
\hline & & & $10^{\circ} \mathrm{C}$ & $5^{\circ} \mathrm{C}$ \\
\hline \multirow{3}{*}{$R_{c}{ }^{(0)}$} & Experiment & 2306 & 2166 & 3320 \\
\hline & Approximate Sol. & 2550 & 2669 & 2985 \\
\hline & SUPORE & 2601 & 2673 & 2940 \\
\hline \multirow{3}{*}{$\tau / \tau_{K}$} & Experiment & 0,507 & 0,401 & 0,362 \\
\hline & Approximate Sol. & 0,432 & 0,381 & 0,352 \\
\hline & SUPORE & 0,442 & 0,399 & 0,376 \\
\hline
\end{tabular}

Table 2. 


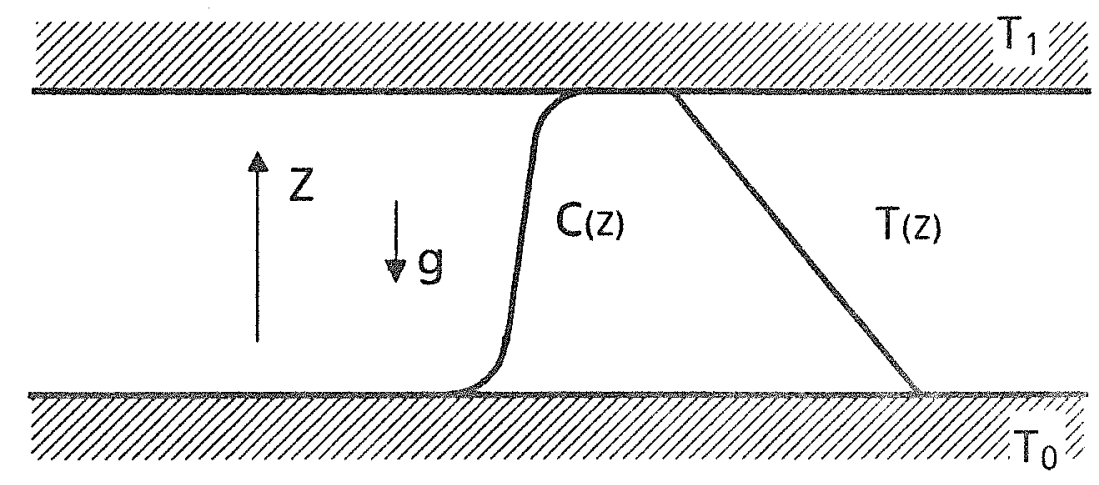

Fig. 1 


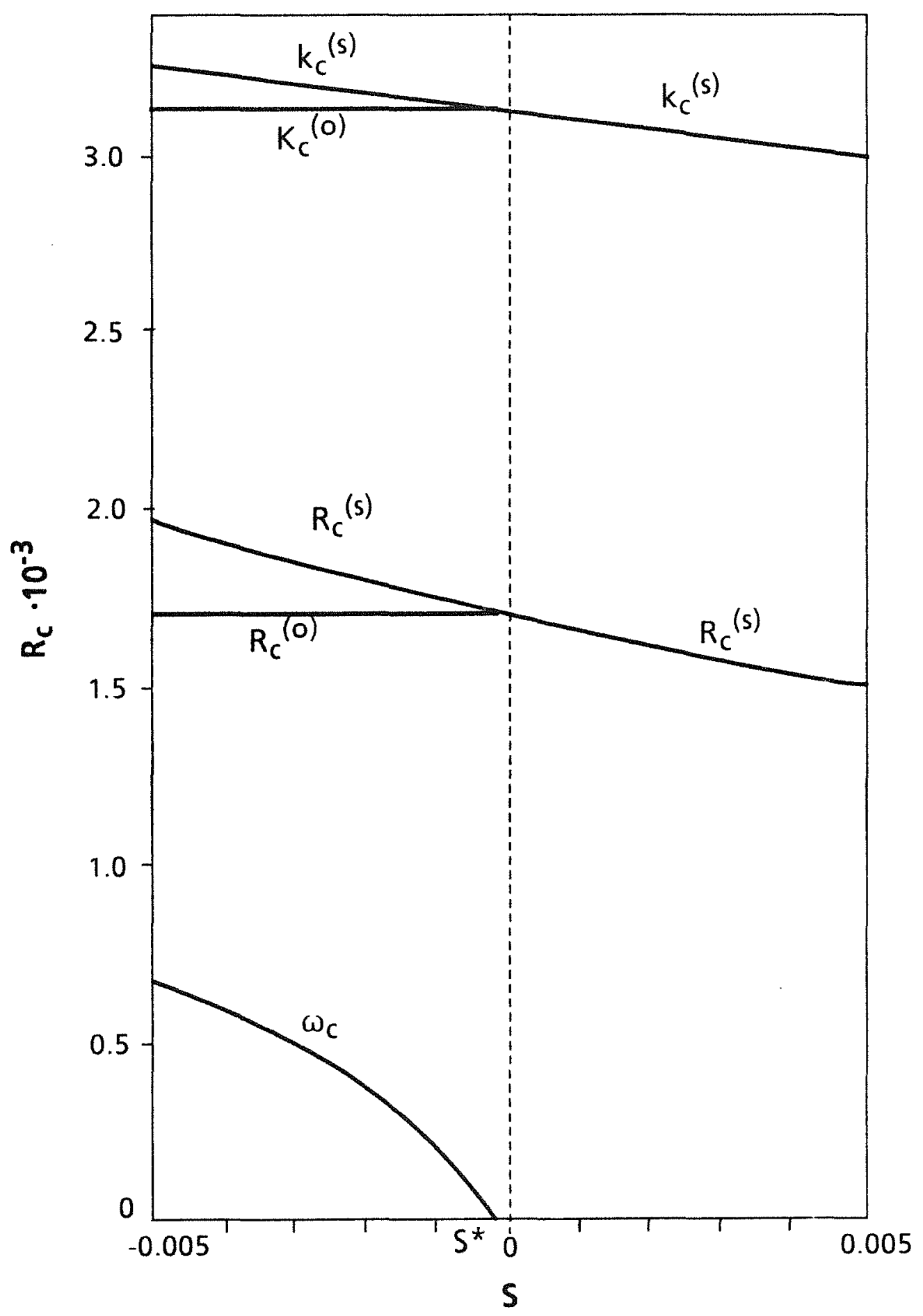

Fig. 2 


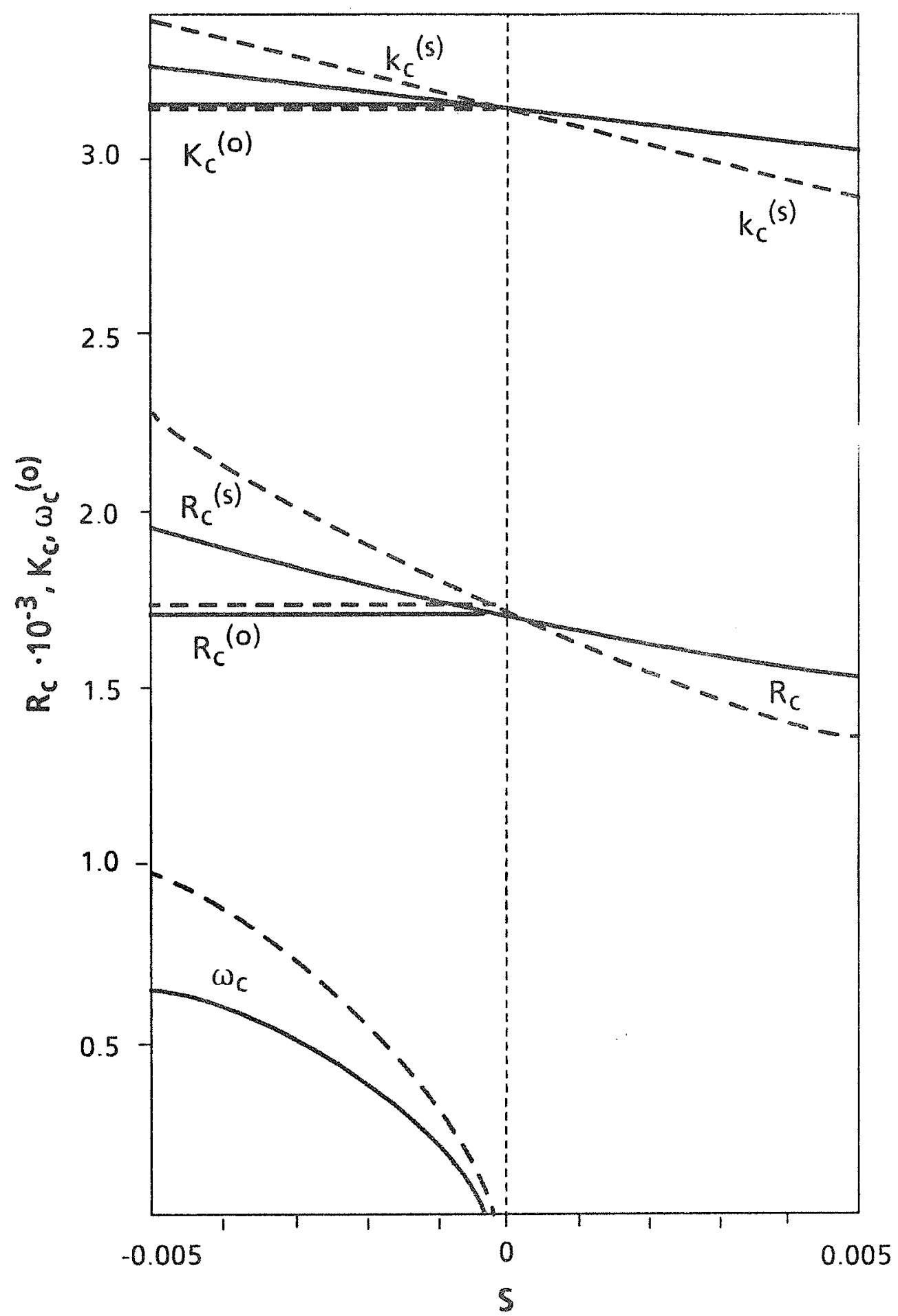

Fig. 3 


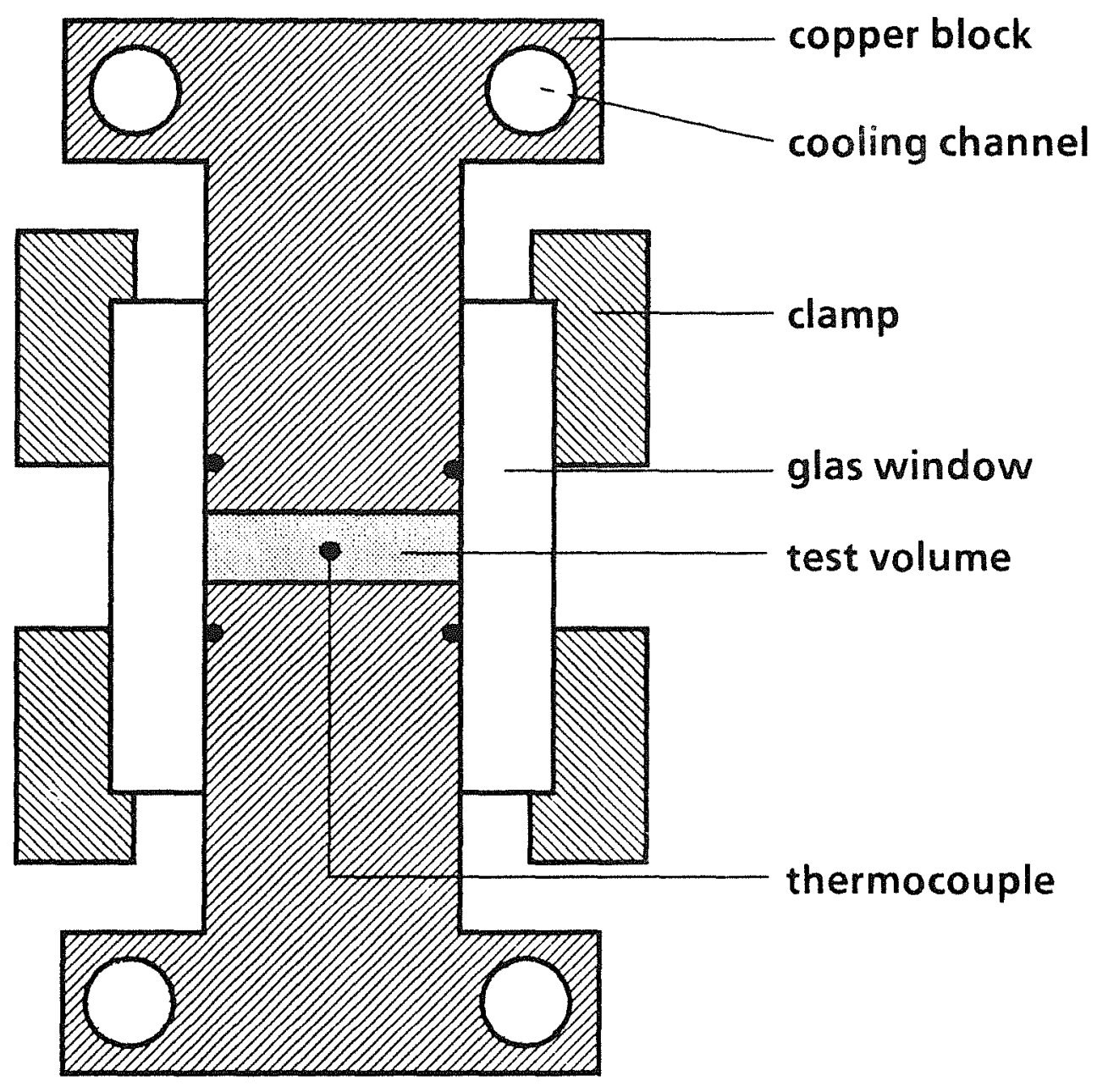

Fig. 4 


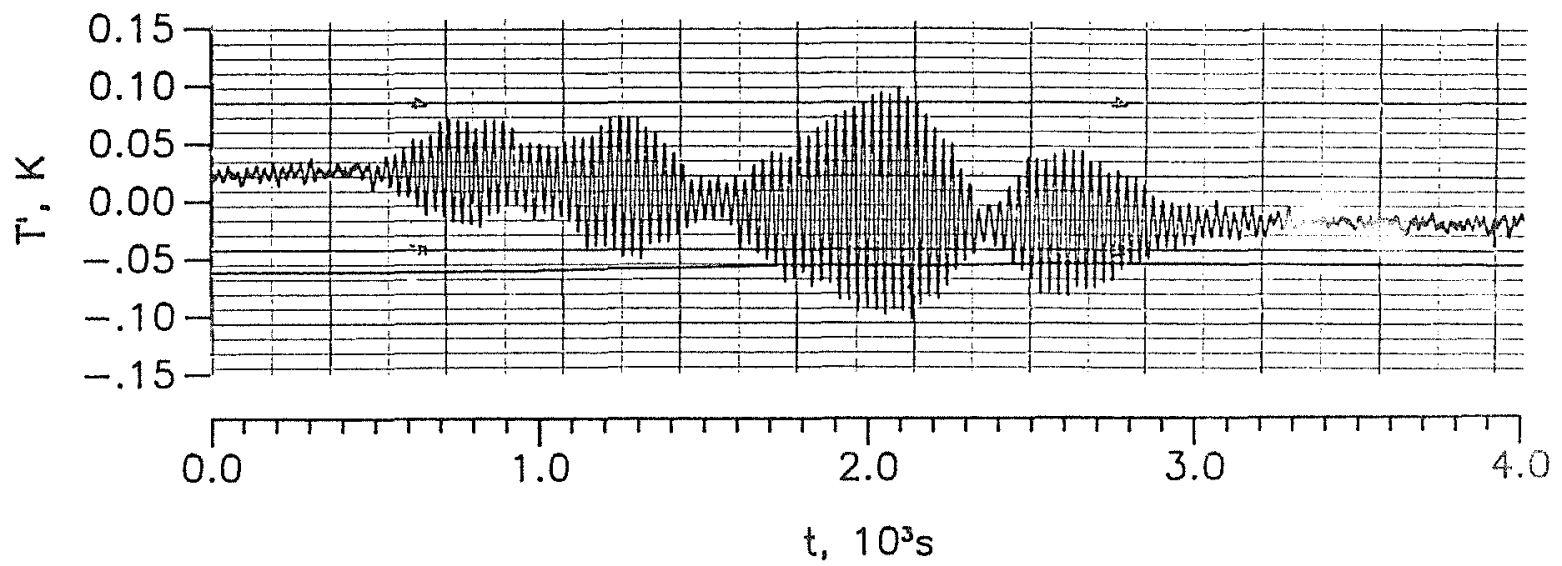

Fig. 5 

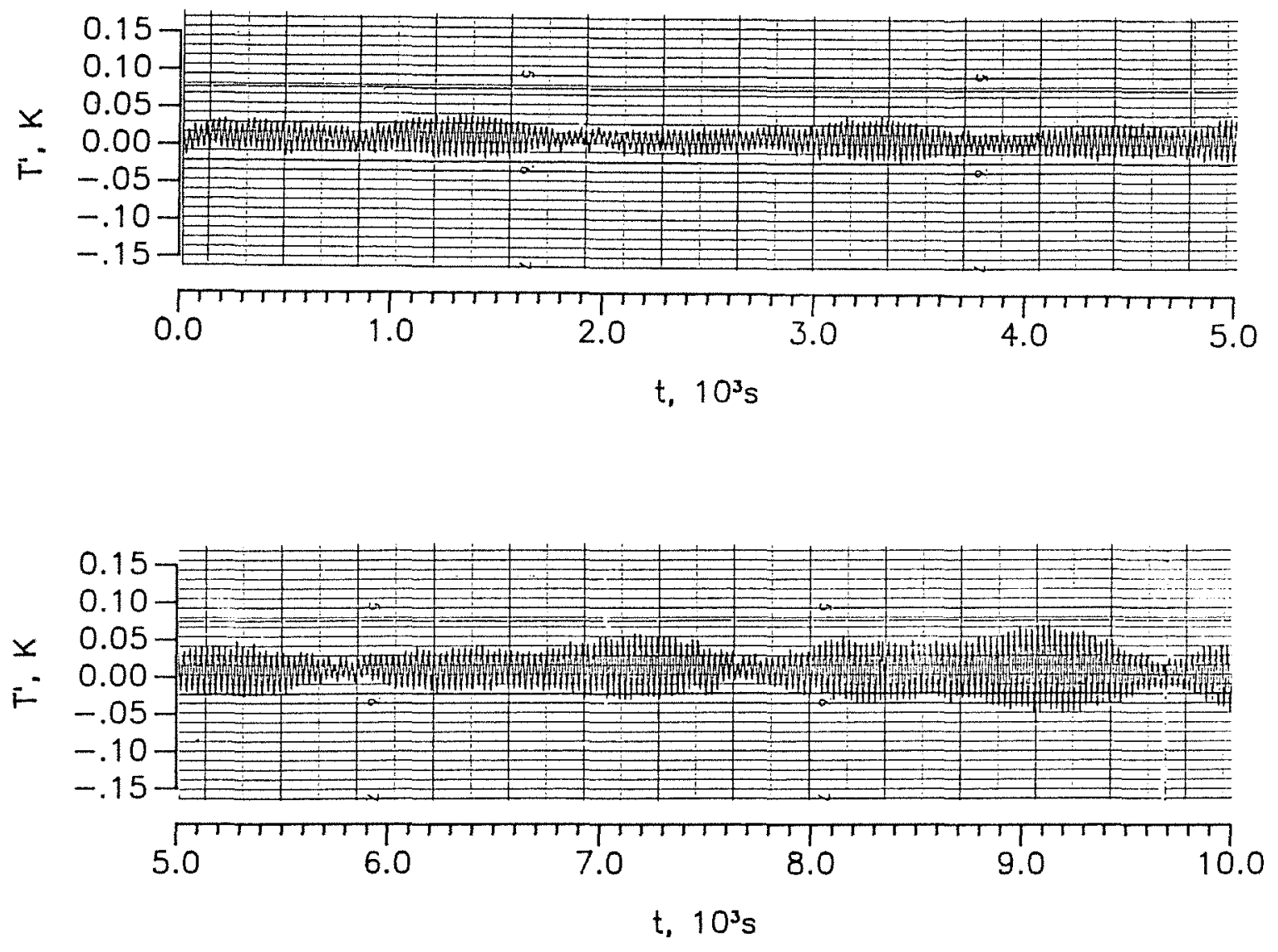

Fig. 6 


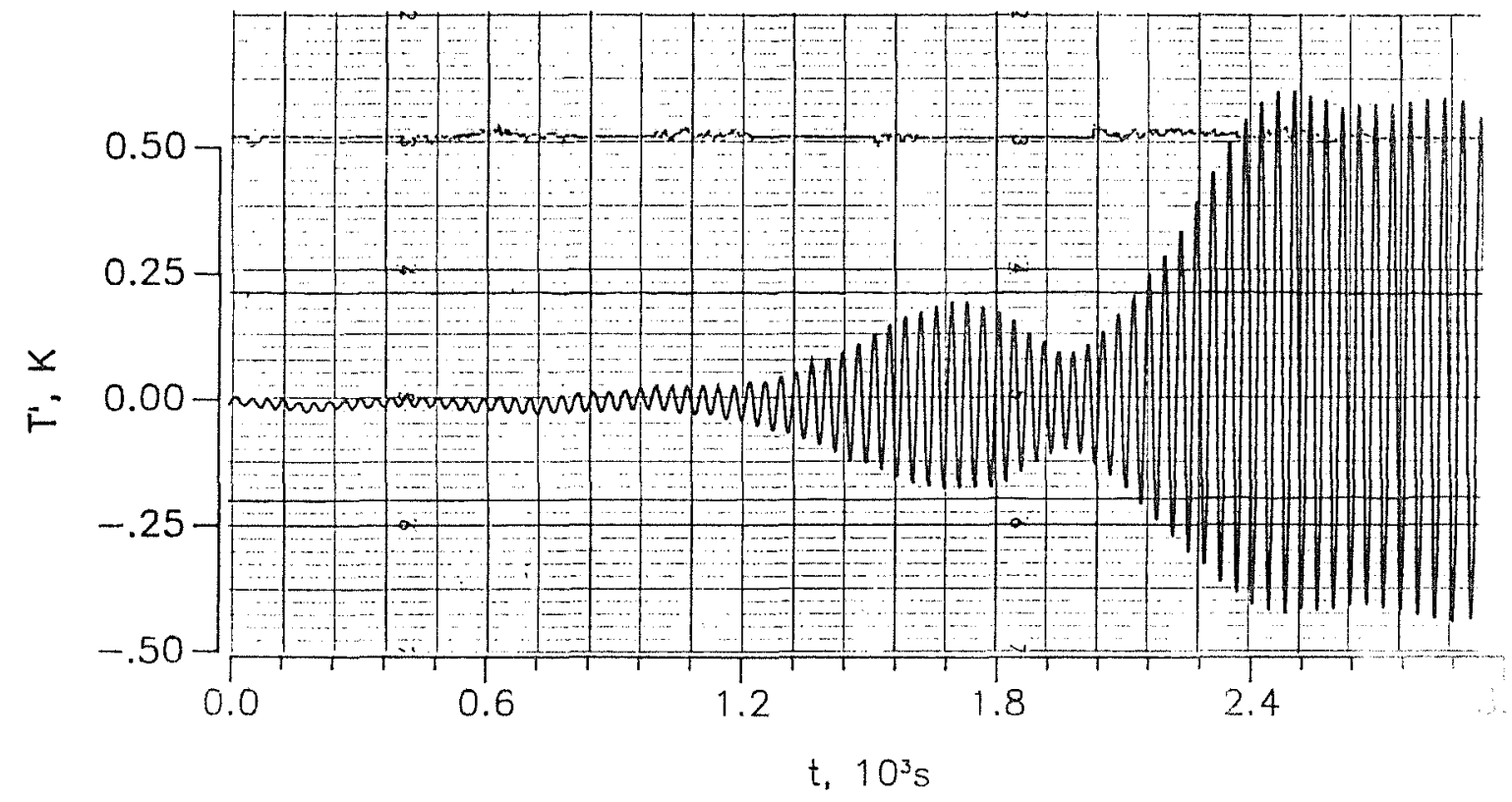

Fig. 7 

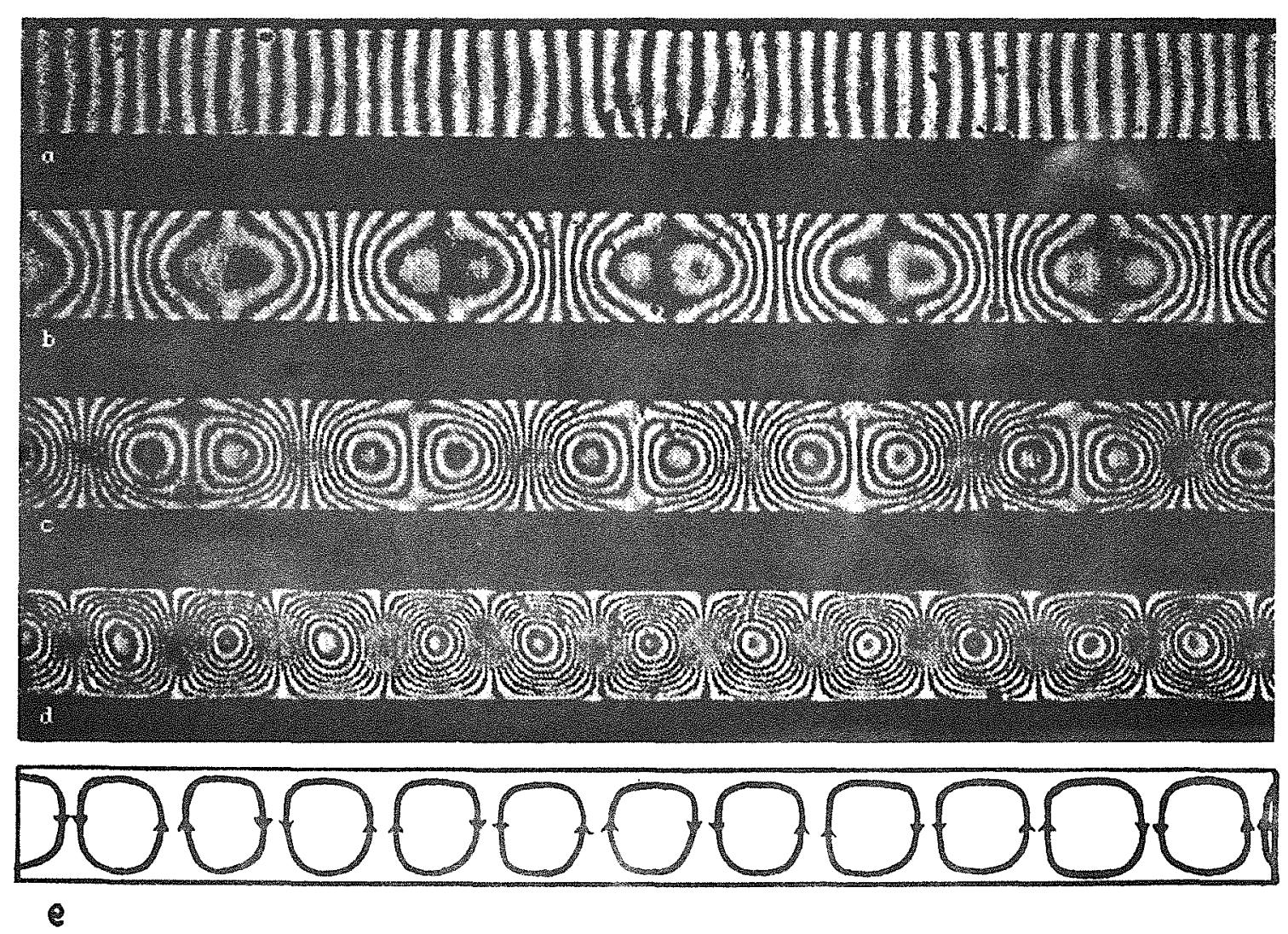

Fig. 8 
Zait $t^{\circ} s$

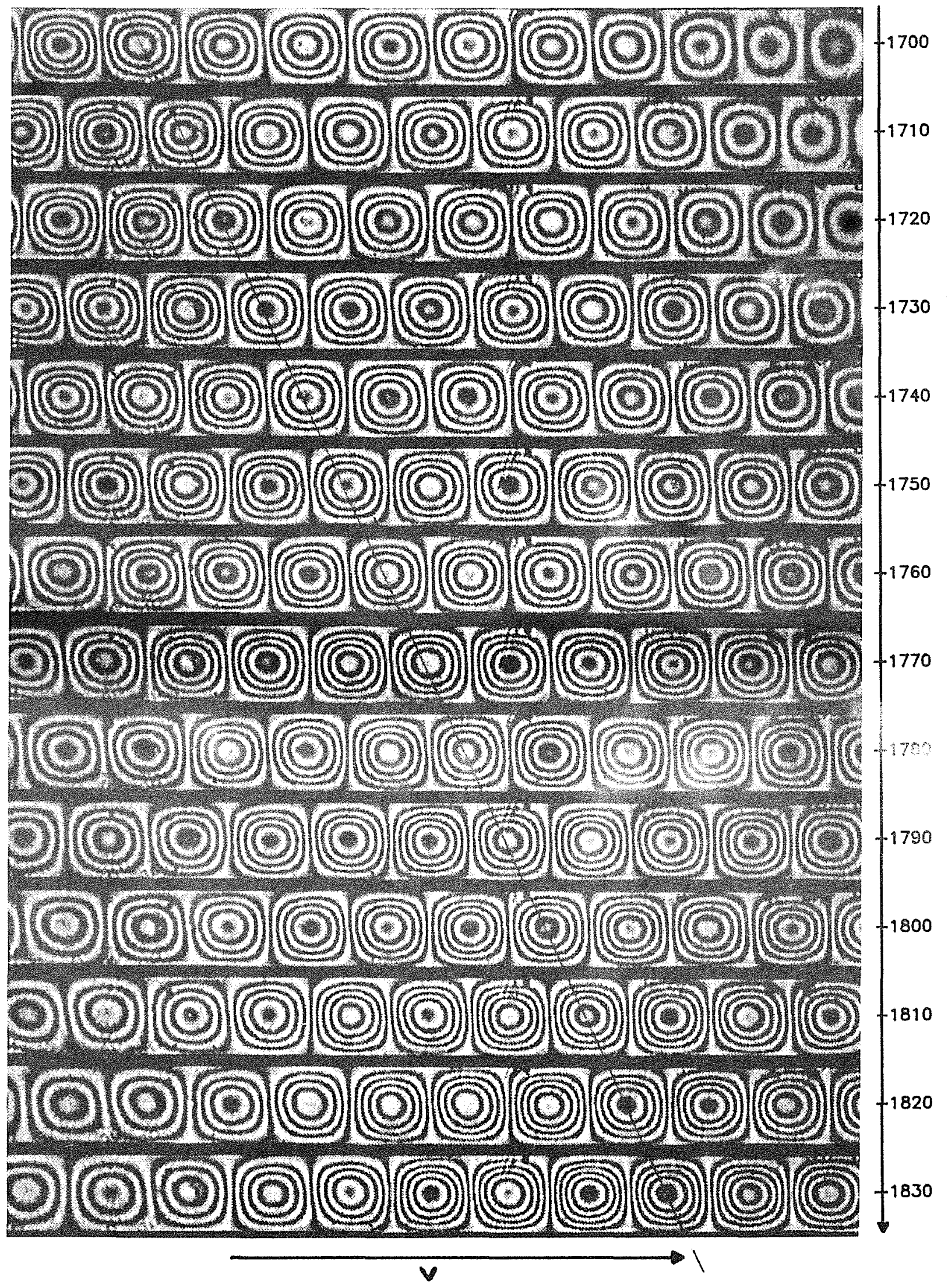

Fig. 9 

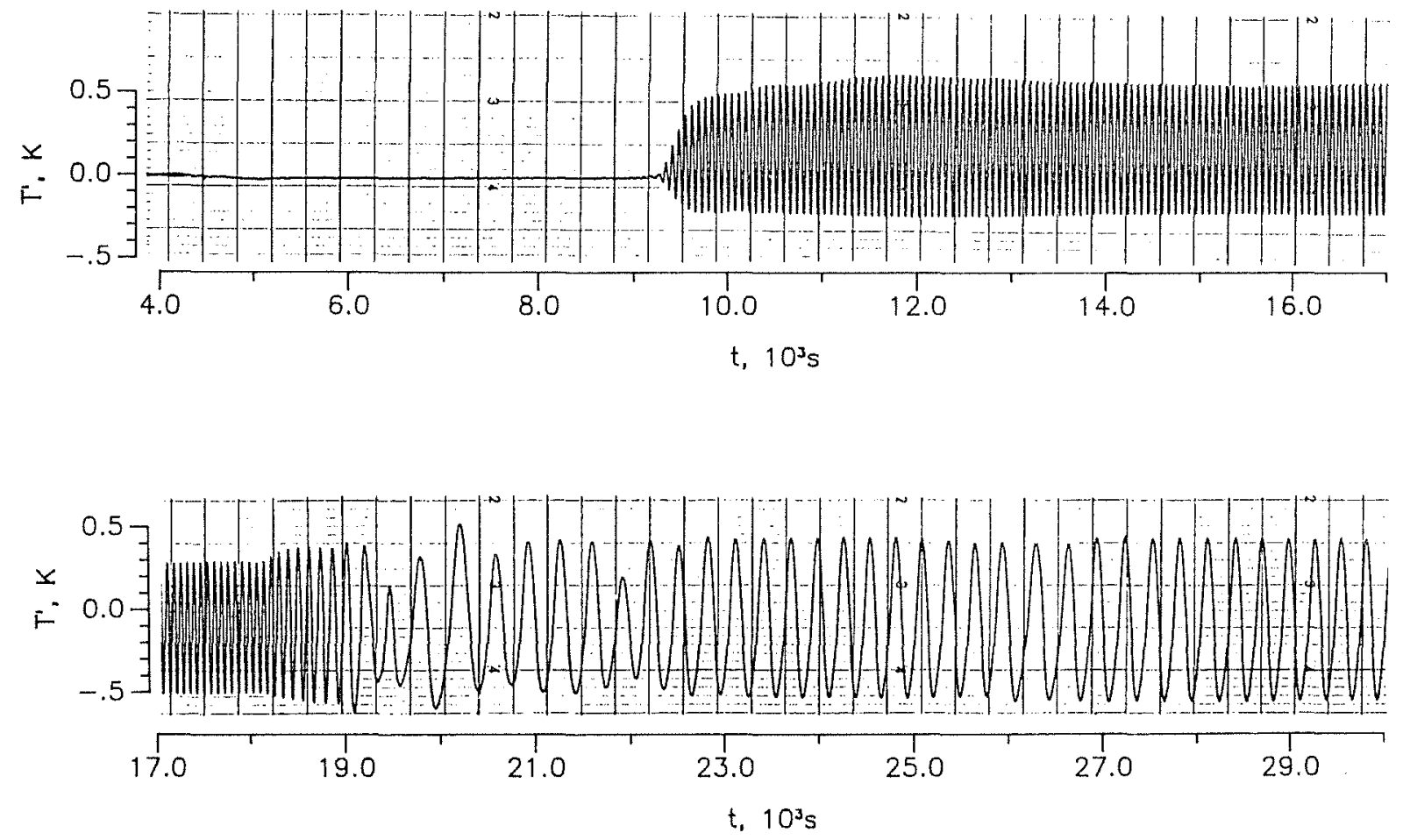

Fig. 10 

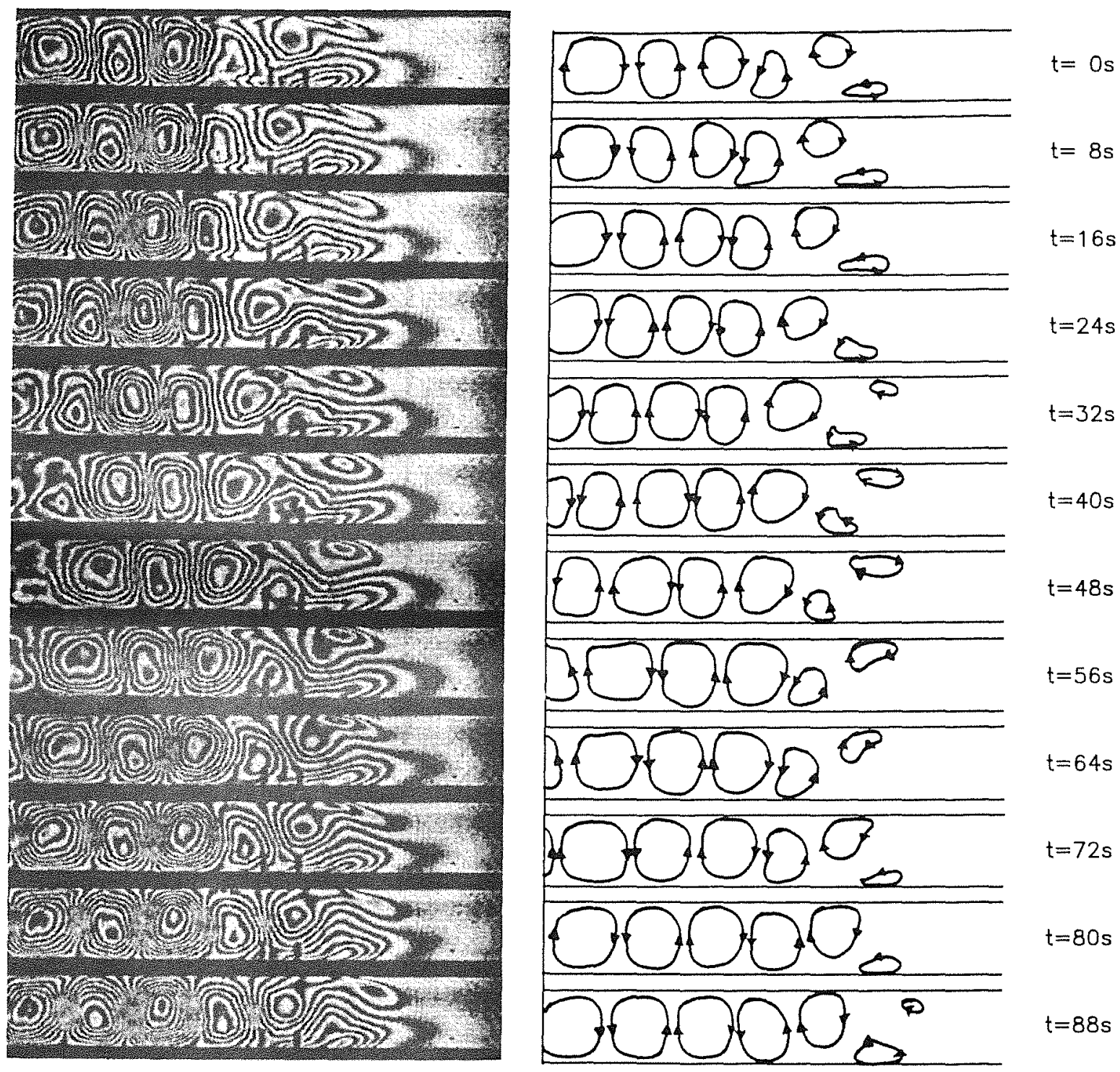

Fig. 11 


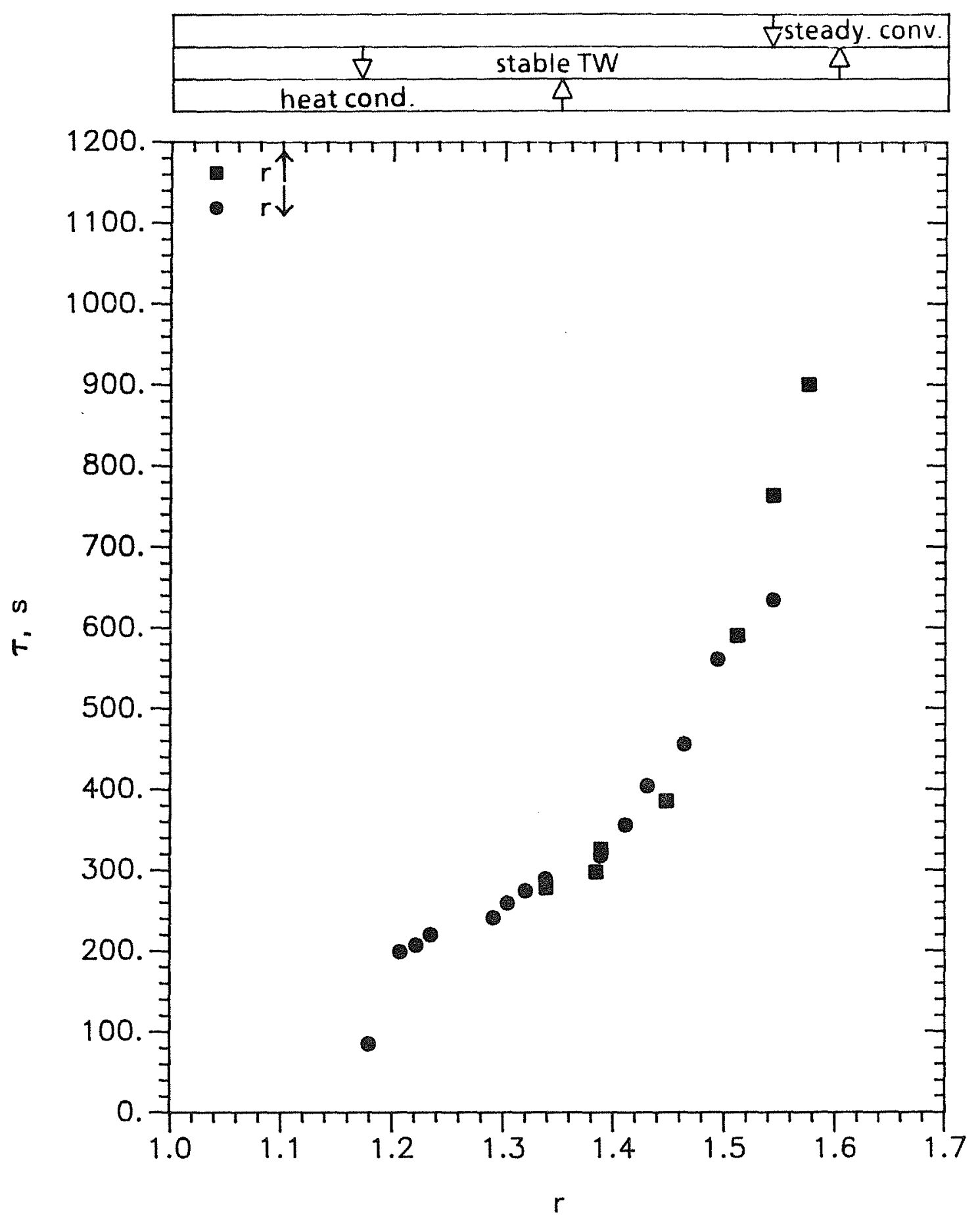

Fig. 12 


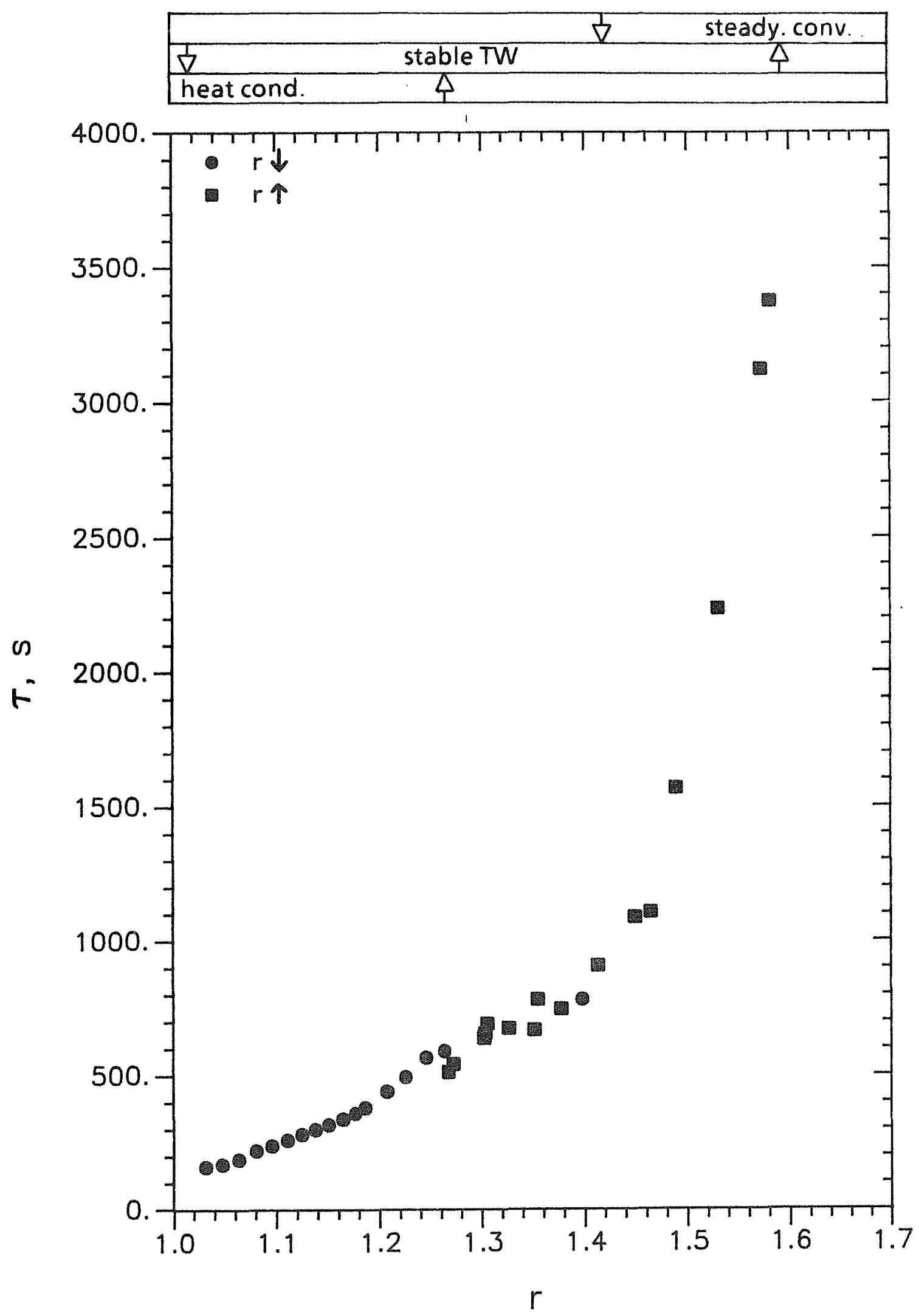

Fig. 13 


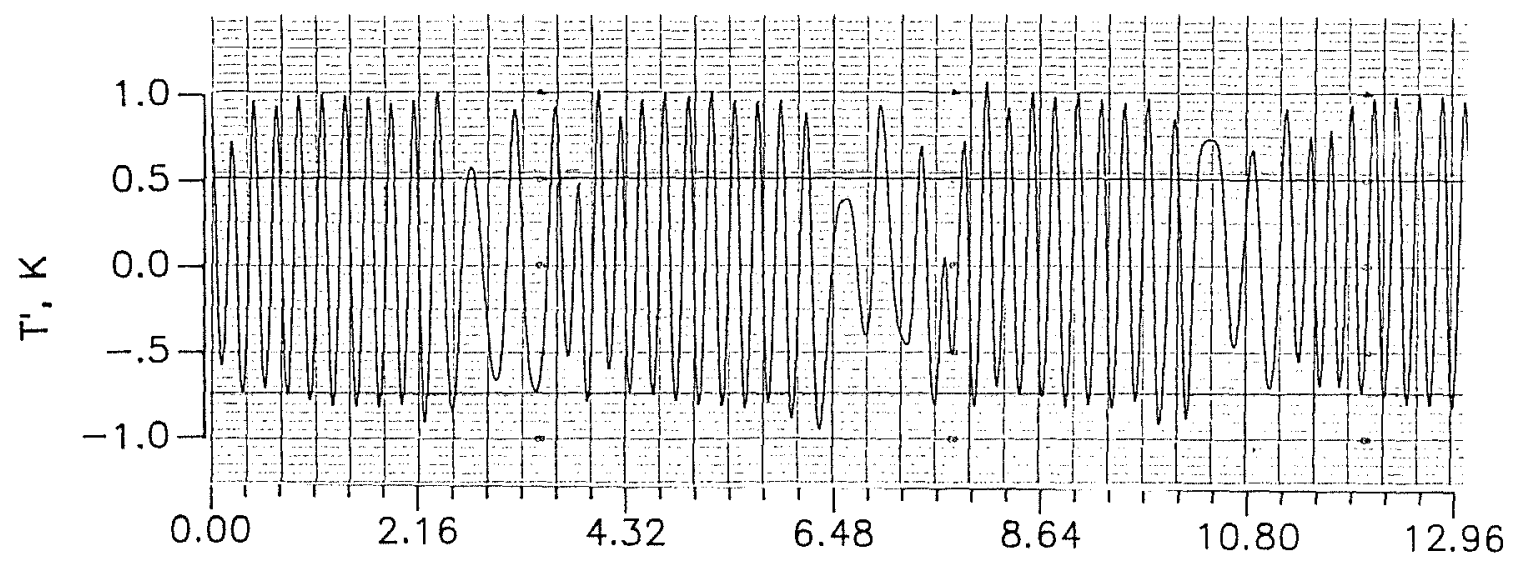

a)

$t, 10^{3} \mathrm{~s}$

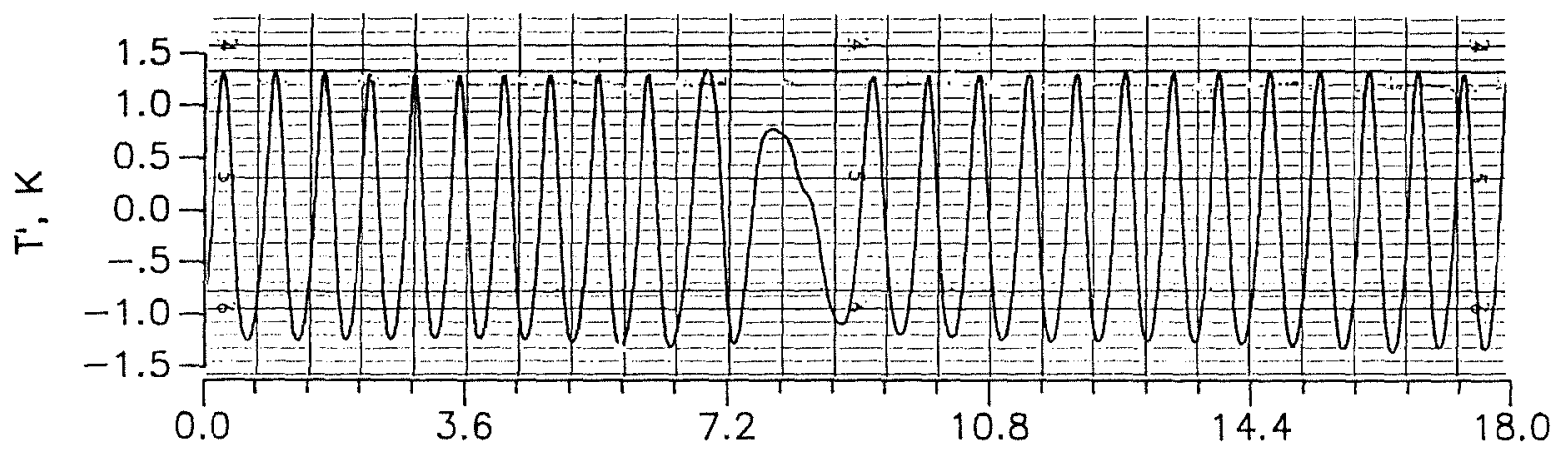

b)

$t, 10^{3} \mathrm{~s}$

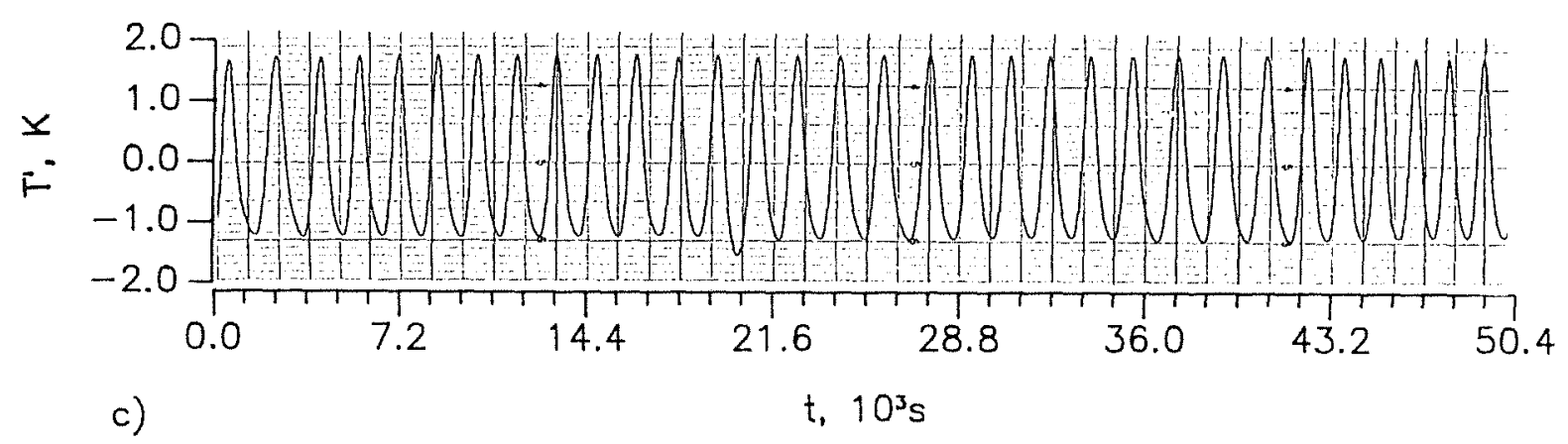


a)

\begin{tabular}{|c|c|}
\hline 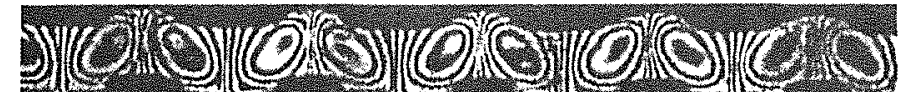 & $t=0 s$ \\
\hline 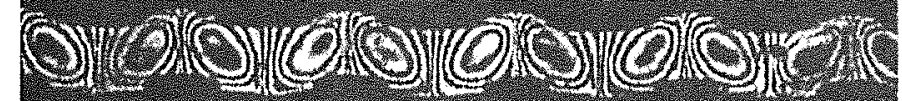 & $t=60 \mathrm{~s}$ \\
\hline & $t=120 \mathrm{~s}$ \\
\hline & $t=180 \mathrm{~s}$ \\
\hline 8) $)(6$ & \\
\hline
\end{tabular}

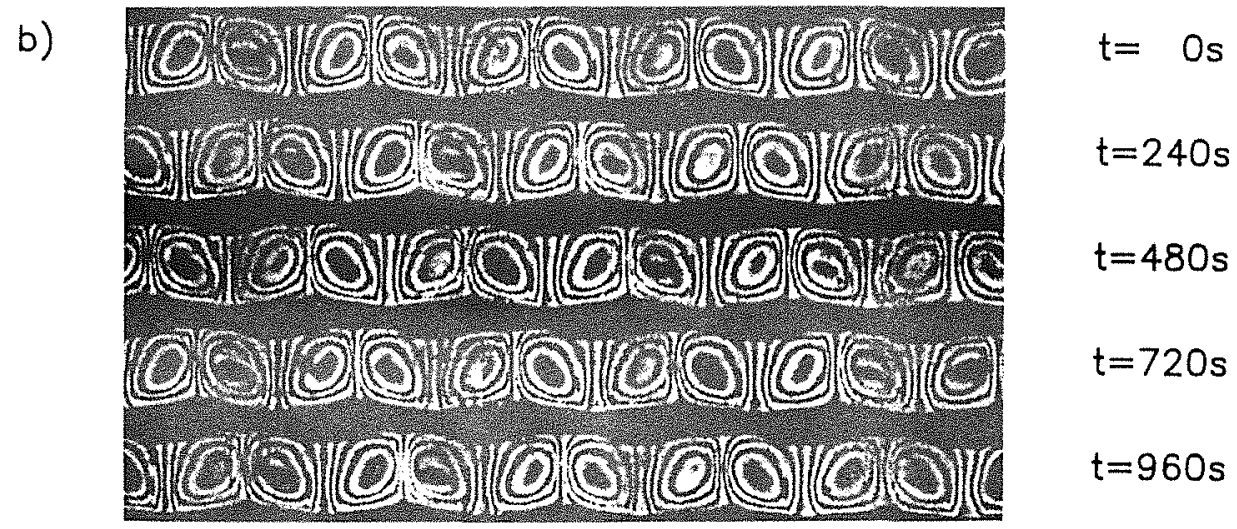

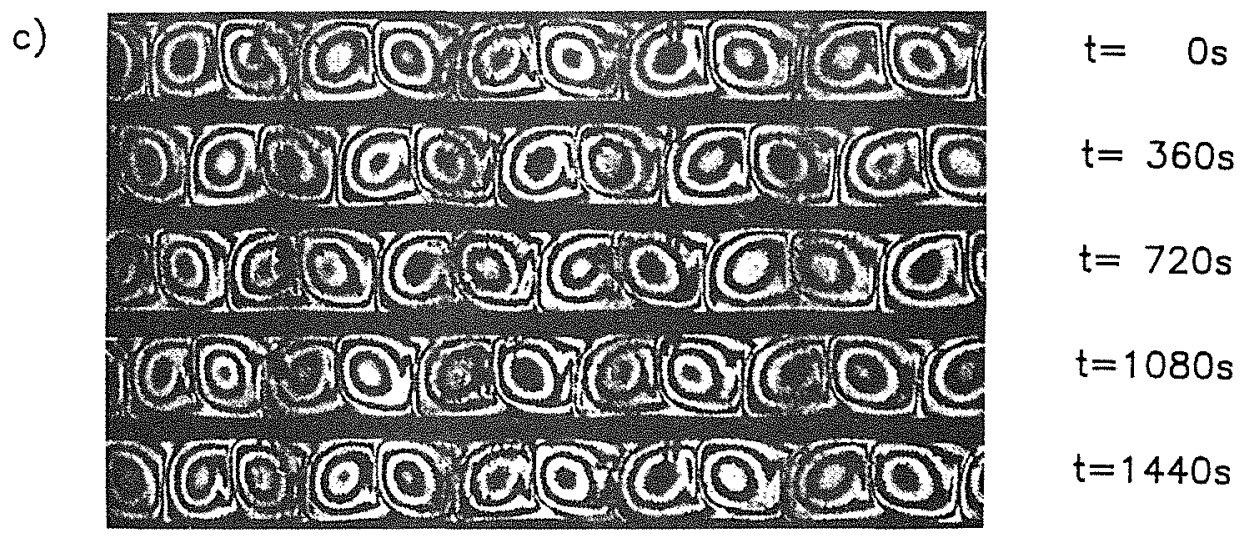

Fig. 15 


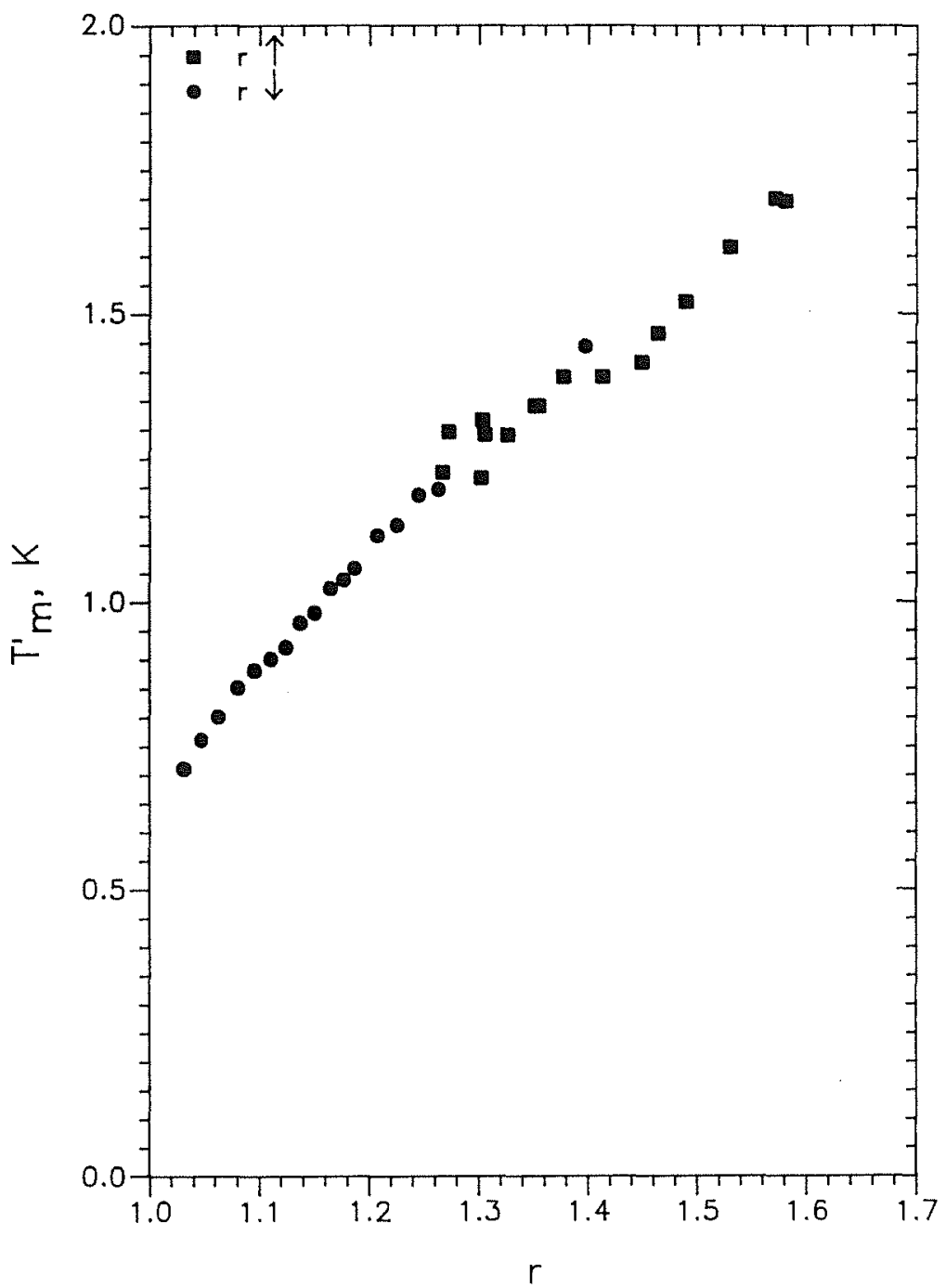

4.12

Fig. 16 


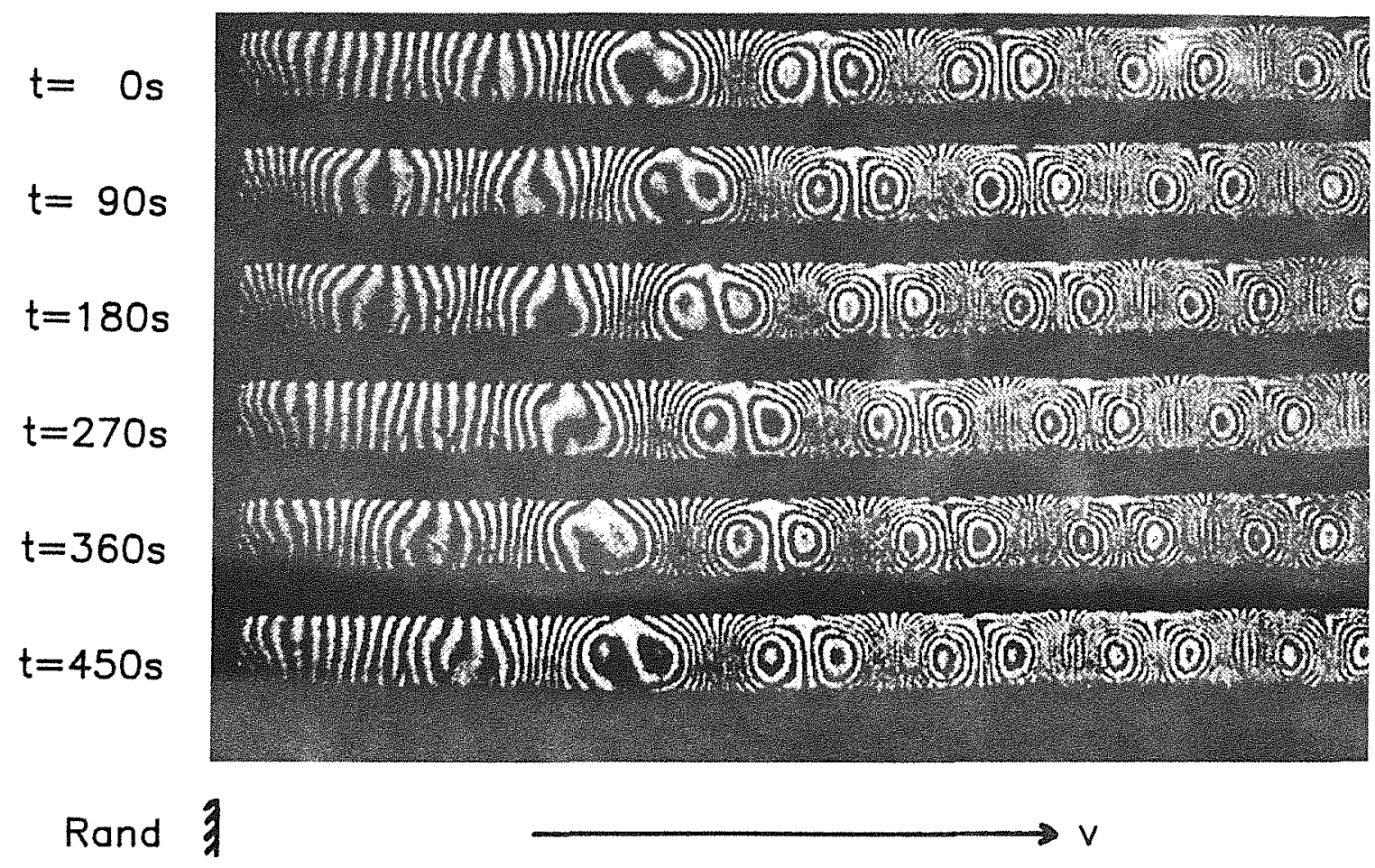

Fig. 17 


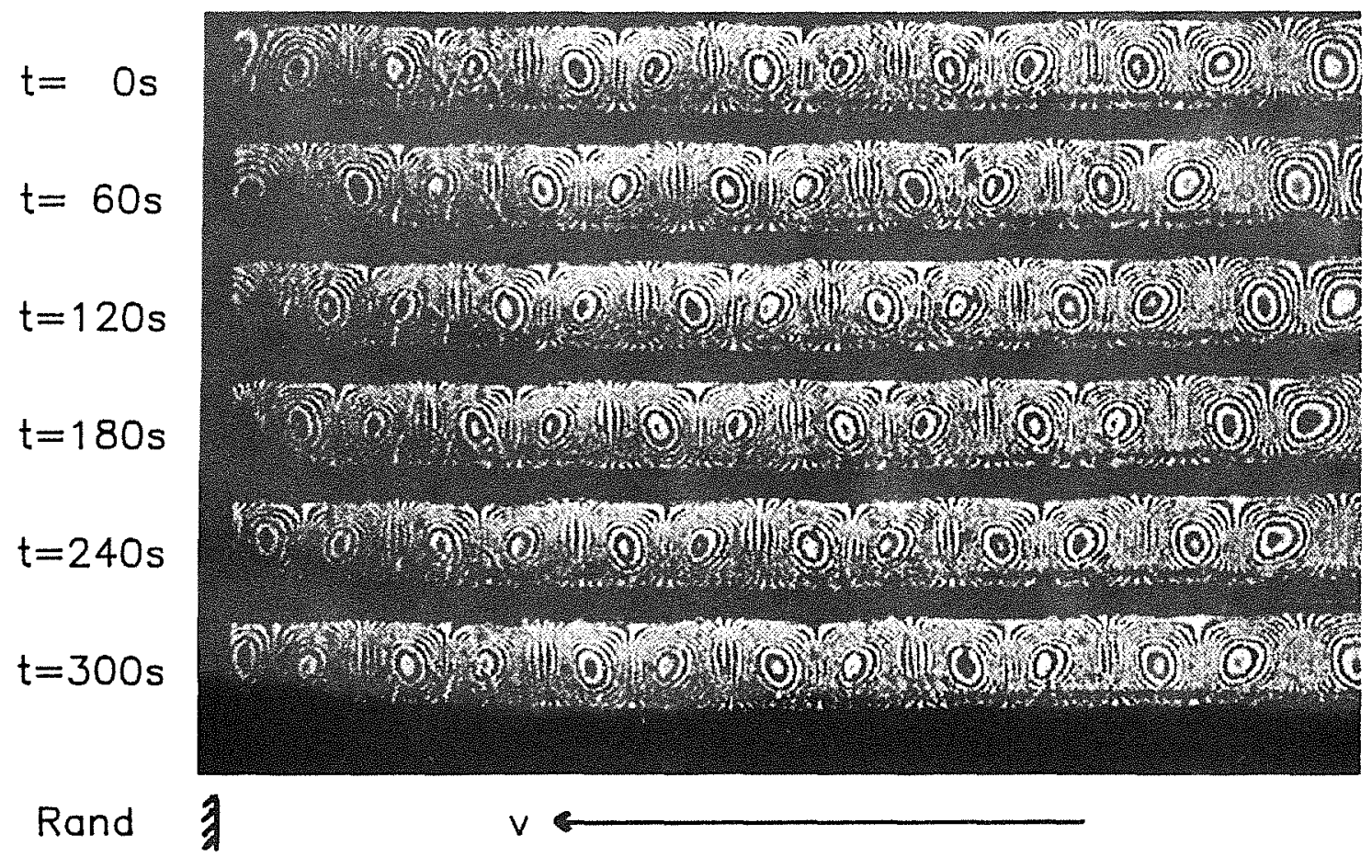

Fig. 18 

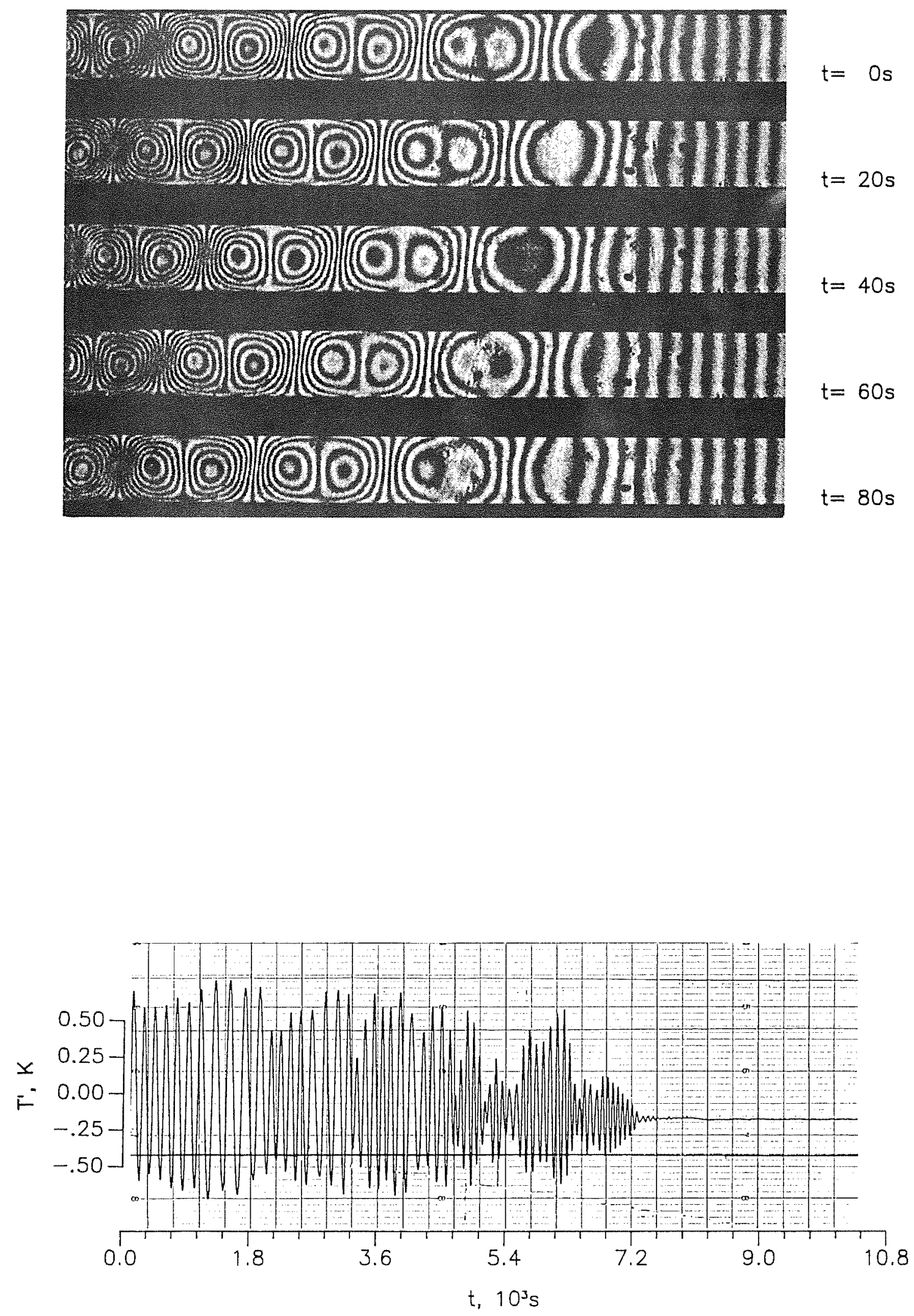

Fig. 19 

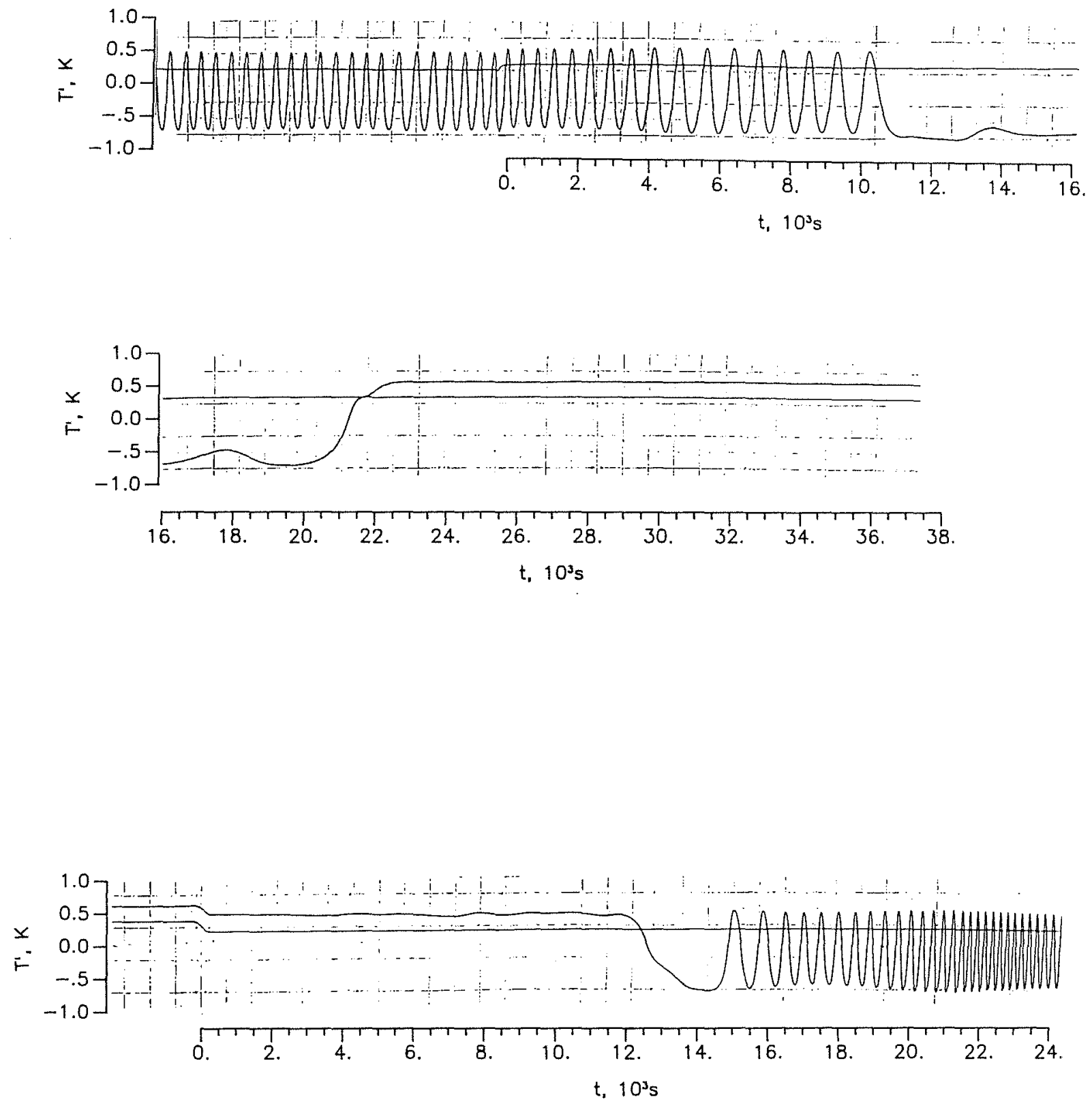

Fig. 20 